\title{
Weak lensing density profiles and mass reconstructions of the galaxy clusters Abell 1351 and Abell 1995^
}

\author{
K. Holhjem ${ }^{1,2}$, M. Schirmer ${ }^{1,3}$, and H. Dahle ${ }^{2}$ \\ 1 Argelander-Institut für Astronomie (AIfA), Universität Bonn, Auf dem Hügel 71, 53121 Bonn, Germany \\ e-mail: kholhjem@astro.uni-bonn.de \\ 2 Institute of Theoretical Astrophysics, University of Oslo, PO Box 1029 Blindern, 0315 Oslo, Norway \\ ${ }^{3}$ Isaac Newton Group of Telescopes, Calle Alvarez Abreu 70, 38700 Santa Cruz de La Palma, Spain
}

Received 6 November 2007 / Accepted 11 June 2009

\begin{abstract}
Aims. The aim of the present work is to study the overall mass distribution of the galaxy clusters Abell 1351 and Abell 1995 using weak gravitational lensing. These clusters have a very different mass structure and dynamical state and are the two extremes from a larger sample of $38 \mathrm{X}$-ray luminous clusters of similar size and redshift.

Methods. We measure the shear values of faint background galaxies and correct for PSF anisotropies using the KSB+ method. Twodimensional mass maps of the clusters are created using a finite-field mass reconstruction algorithm, and verified with aperture mass statistics. The masses inferred from the reconstructions are compared to those obtained from fitting spherically symmetric SIS- and NFW-models to the tangential shear profiles. We discuss the NFW concentration parameters in detail.

Results. From the mass reconstructions we infer $M_{2 \mathrm{D}}\left(<r_{200}\right)$-masses of $11.7 \pm 3.1 \times 10^{14} h_{70}^{-1} M_{\odot}$ and $10.5 \pm 2.7 \times 10^{14} h_{70}^{-1} M_{\odot}$ for Abell 1351 and Abell 1995, respectively. About 3' northeast of the main mass peak of Abell 1351, we detect a significant secondary peak both in the mass reconstruction and from aperture mass statistics. This feature is also traced by cluster members selected by means of their $V-I$ colour, and is therefore likely a real substructure of Abell 1351. From our fits to the tangential shear we infer masses of the order of $M_{200} \sim 8-9 \times 10^{14} h_{70}^{-1} M_{\odot}$ (Abell 1351) and $M_{200} \sim 5-6 \times 10^{14} h_{70}^{-1} M_{\odot}$ (Abell 1995). The concentration parameters remain poorly constrained by our weak lensing analysis.
\end{abstract}

Key words. gravitational lensing - cosmology: dark matter - galaxies: clusters: individual: Abell 1351, Abell 1995

\section{Introduction}

Comprising the most massive gravitationally bound structures in the Universe, galaxy clusters are essential in providing a deeper understanding of the properties of dark matter. The recent papers covering 1E 0657-558 (the bullet cluster) by Clowe et al. (2006a) and Abell 520 by Mahdavi et al. (2007) demonstrate the importance of gravitational lensing for understanding the matter content of our Universe. Lensing studies of galaxy clusters provide a powerful way to identify high density peaks in the Universe, independent of their baryonic content (Maturi et al. 2007; Miyazaki et al. 2007; Gavazzi \& Soucail 2007; Schirmer et al. 2007; Wittman et al. 2006; Clowe et al. 2006b; Dahle et al. 2003; Schneider 1996).

Neither the nature nor the dynamical state of the gravitating matter affect the mass estimates obtained through gravitational lensing. These mass measurements are only changed by gravitation and the geometrical configuration between observer, lens, and source. Although this makes lensing a unique tool, such measurements can be biased by e.g. different mass concentrations along the line-of-sight.

In this paper we analyse two clusters of galaxies at intermediate redshift $(z=0.32)$, Abell 1351 and Abell 1995. These clusters are selected from a lensing study of 38 highly X-ray

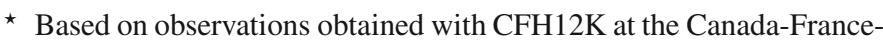
Hawaii Telescope (CFHT) which is operated by the National Research Council of Canada, the Institut des Sciences de l'Univers of the Centre National de la Recherche Scientifique and the University of Hawaii.
}

luminous galaxy clusters (Dahle et al. 2002). They were chosen for further investigation because they represent the two extremes in this cluster sample, regarding mass distribution and dynamical state. Re-observing the clusters with the wide-field camera CFH12K at the Canada-France-Hawaii telescope (CFHT) provided a larger field of view $(42.2 \times 28$.'1) than employed by Dahle et al. (2002) $(18.8 \times 18.8)$. This allows us to map the clusters to larger radii than previously possible.

The KSB+ method (Kaiser et al. 1995; Luppino \& Kaiser 1997; Hoekstra et al. 1998) is used to recover the shear values of faint background galaxies in the images. Using a finite-field reconstruction technique (Seitz \& Schneider 2001), we derive two-dimensional mass maps, visualising the surface mass distributions of Abell 1351 and Abell 1995. We also apply aperture mass statistics (Schneider 1996; Schirmer et al. 2007) to our data, comparing the results to confirm mass peak detections. Finally, by fitting predicted shear values from theoretical models to the shapes of the lensed galaxies we estimate the cluster masses. We assume the clusters to be spherically symmetric and to have density profiles following either a singular isothermal sphere (SIS) or a Navarro, Frenk, \& White (1997, 1995; NFW) model.

Data processing and analysis are carried out using mainly the IMCAT software package ${ }^{1}$, Kaiser's July 2005 version for Macintosh. IMCAT is a tool specially designed for weak lensing

\footnotetext{
1 IMCAT is developed by Nick Kaiser (kaiser@hawaii.edu), http://www.ifa.hawaii.edu/ kaiser/imcat/
} 
purposes, and is optimised for shape measurements of faint galaxies. It processes both FITS files and object catalogues.

The outline of this paper is as follows. In Sect. 2 we summarise the observations and software used for our study, in addition to the data reduction. In Sect. 3 we describe the shear reconstruction, focusing on shape estimates and point spread function (PSF) corrections. In Sect. 4 we present the clusters' surface mass density maps and attempt to verify the detected mass peaks using $V-I$ colours and aperture mass statistics. By comparing the measured shear profiles to theoretical expectations, we model the lensing data in Sect. 5. Finally we present and discuss our results in Sect. 6, and our conclusions in Sect. 7.

Throughout this paper we assume a $\Lambda$ CDM cosmology, with $\Omega_{\mathrm{M}}=0.3, \Omega_{\Lambda}=0.7$, and $h_{70}=H_{0} /\left(70 \mathrm{~km} \mathrm{~s}^{-1} \mathrm{Mpc}^{-1}\right)$. Errors are given on the $1 \sigma$ level.

\section{Observations and data reduction}

\subsection{Data acquisition}

The galaxy clusters Abell 1351 and Abell 1995 are centred at the positions $11^{\mathrm{h}} 42^{\mathrm{m}} 30^{\mathrm{s}} .7+58^{\circ} 32^{\prime} 21^{\prime \prime}$ and $14^{\mathrm{h}} 52^{\mathrm{m}} 50^{\mathrm{s}} .4+58^{\circ} 02^{\prime} 48^{\prime \prime}$, respectively. They were observed with the CFHT on the 4 nights of 7-11 May, 2000, using the wide-field CCD mosaic camera CFH12K. A total exposure time of $5400 \mathrm{~s}$ was obtained for both clusters in $I$ band. However, due to seeing $>1^{\prime \prime}$, we rejected 3 exposures from the Abell 1351 data set, resulting in $3600 \mathrm{~s}$ for this cluster. This corresponds to a $5 \sigma$ limiting magnitude of $I=25.2 \pm 0.1$ for point sources in both pointings. The seeing in the final coadded images equals 0.95 and 1'. 15 for Abell 1351 and Abell 1995, respectively. The number density of the lensed background galaxies is $16 \operatorname{arcmin}^{-2}$ for both clusters, and the ellipticity dispersion (after PSF correction) for each component is $\sigma_{g}=0.43$ and $\sigma_{g}=0.51$ for Abell 1351 and Abell 1995, respectively. The larger dispersion for Abell 1995 is explained by the 20\% larger image seeing, which enlarges the PSF correction factors and their uncertainties.

The $\mathrm{CFH} 12 \mathrm{~K}$ mosaic camera covers a field of $12 \mathrm{k} \times 8 \mathrm{k}$ pixels in total, representing an area of $42 ! 2 \times 28$ '. 1 on the sky. The pixel scale is $00^{\prime} .206$ when mounted at the CFHT prime focus.

In addition we made use of the $V$-band data obtained by Dahle et al. (2002) to verify neighbouring peaks present in our two-dimensional mass maps described in Sect. 4. These data were obtained at the $2.24 \mathrm{~m}$ University of Hawaii telescope using the UH8K mosaic camera, covering an area of $4 \mathrm{k} \times 4 \mathrm{k}$ pixels (rebinned $2 \times 2$ ) mapping 18'.8 $\times 18$ '.8 of the sky. Each image has a total exposure time of $12600 \mathrm{~s}$, resulting in a depth comparable to our $I$-band data, with $5 \sigma$ limiting magnitudes of $V=25.9 \pm 0.1$ and $V=25.8 \pm 0.1$ for Abell 1351 and Abell 1995, respectively. Further details about the reduction process and coaddition of the $V$-band data can be found in Dahle et al. (2002).

As Abell 1351 and Abell 1995 are both located at redshift $z=0.32$, they have a similar correspondence between physical and angular scale, given as $1 \mathrm{Mpc}=215^{\prime \prime}$.

\subsection{Image processing}

\subsubsection{Pre-processing}

To remove the bias level in each frame we used the mean value of the overscan region from the corresponding chip. The flatfielding was carried out using a master night time flat, made from averaging 56 night time exposures; most of them so-called "blank" fields and all well displaced from each other. The fringing that occurs in $I$-band exposures is also captured in this type of flat, and upon dividing the object exposures by it the fringes were cleanly removed. To estimate the background level in the exposures, we used the heights of the minima of the sky level present to create a model for each individual frame. After subtraction the median sky level was set to zero.

As fringing is an additive effect and not a multiplicative one, ideally the fringes should be subtracted. Since we had no twilight flats available, standard defringing could not be performed. The photometric error introduced by division is negligible, as the amplitude of the fringes compared to the sky background after flatfielding was of the order of $2 \%$. However, since fringing acts mostly on small angular scales, its treatment will affect the shapes of the small and faint background galaxies used for weak lensing. To investigate this we obtained a set of 10 archival images of the Deep3 field (Hildebrandt et al. 2006), taken with the Wide Field Imager at the $2.2 \mathrm{~m} \mathrm{MPG/ESO} \mathrm{tele-}$ scope through their I-band filter. As the Deep3 field does not contain any massive clusters it is very well suited for this test. Two different coadded images were created. In the first case the data were flatfielded using twilight flats, after which a fringing model was created from the flatfielded data and subtracted. The second coadded image was processed in the same way as our CFHT data, i.e. the data were flatfielded and fringe-corrected by division of a night-time flat. We then measured the shapes of a common set of $\sim 12000$ galaxies in both images (see Sect. 3 ) and created two mass reconstructions, using the same technique and smoothing scale as for Abell 1351 and Abell 1995 (see Sect. 4). We found that the rms of the difference of the two mass maps is a factor of 2.5 smaller than the noise of the individual mass maps, mainly caused by the intrinsic ellipticities of background galaxies. The effect in our $\mathrm{CFH} 12 \mathrm{~K}$ data is much smaller, as the CFH12K I-band filter has a blue cut-on at around $730 \mathrm{~nm}$ and a red cut-off at $950 \mathrm{~nm}$. The ESO I-band filter on the other hand opens at $800 \mathrm{~nm}$ and has no cut-off on the red side. Hence the fringing amplitude in the comparison data set from ESO is up to 5 times higher than that from CFHT. We conclude that our analysis of Abell 1351 and Abell 1995 is not affected by our fringe correction.

\subsubsection{Masking}

The CFH12K mosaic contains some bad pixels and columns, in particular two of the CCDs suffer from this. By using Nick Kaiser's ready made CFH12K masks ${ }^{2}$ as global masks, all bad areas were ensured to be ignored. An additional patch of $219 \mathrm{bad}$ columns in CCD00 was also added to the global masks. We did not make further individual masks for each exposure, as most spurious detections were filtered out during the astrometric calibration. Suspicious objects in the final catalogue were in addition rejected by visual examination.

\subsubsection{Astrometric calibration}

Wide-field data typically do not have a simple relation between the sky coordinates and those of the detector. A mapping from pixel coordinates onto a planar projection of the sky needs therefore be performed. We solved for this through a series of steps.

First, all objects in each exposure were detected and aperture photometry carried out. By plotting $r_{g}$ ( $\sim$ half-light radius) vs. instrumental magnitude of the objects in each exposure, we extracted the moderately bright, non-saturated stars suitable for

${ }^{2}$ http://www.ifa.hawaii.edu/ kaiser/cfh12k/masks/ 

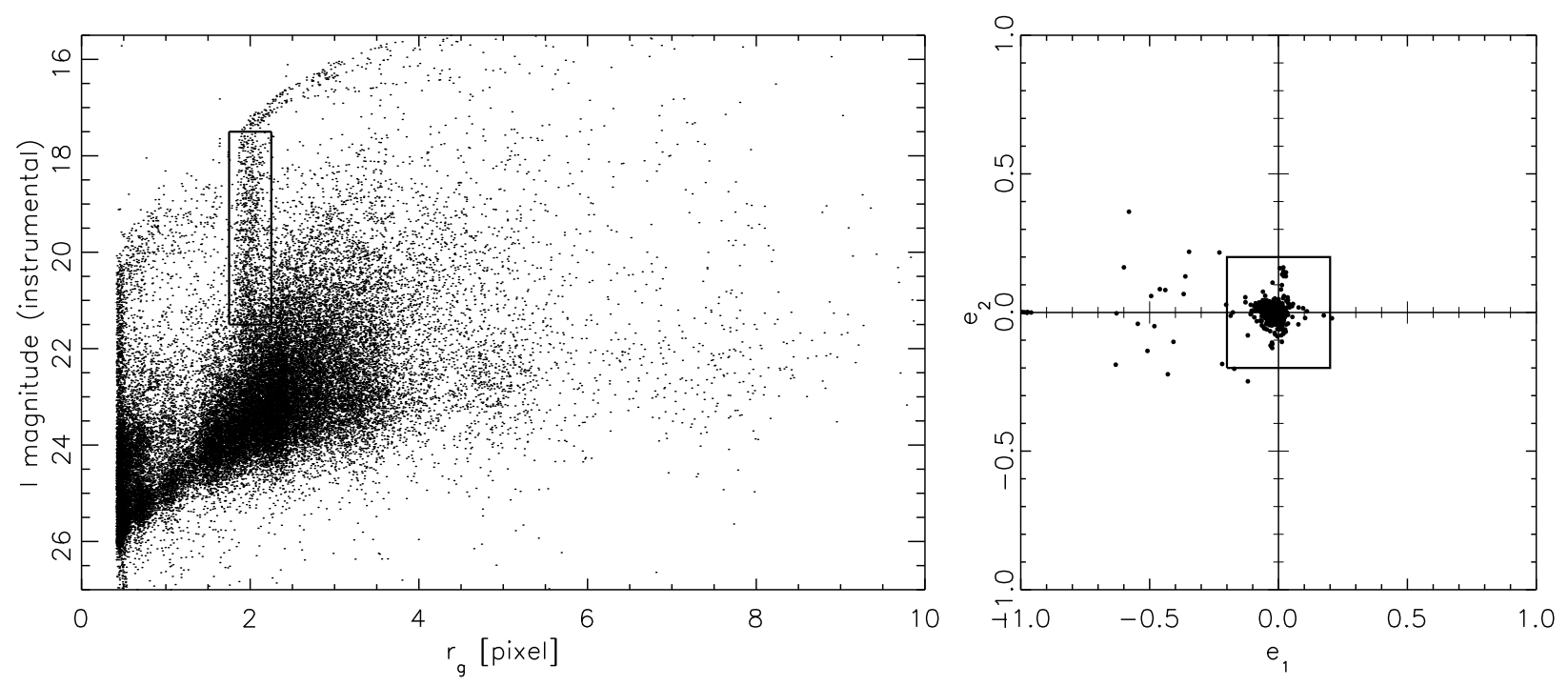

Fig. 1. Example of size-magnitude (left) and weighted ellipticity parameter diagram (right) for an arbitrary exposure. The moderately bright, nonsaturated stars were chosen from within the squares and utilised in the astrometric solution, the right plot containing only the stars chosen from the left plot. The stars utilised in the PSF corrections (Sect. 3.1) were selected the same way.

deriving an astrometric solution. Their weighted ellipticity parameters (defined by Kaiser et al. 1995), $e_{1,2}$, were in addition plotted and eyeballed, and the main clustering of objects were selected to ensure a catalogue containing purely stars (see Fig. 1 for an example). IMCAT only offers squared regions for extracting objects from a two-dimensional parameter space. Although a circle would be a more natural choice for the right panel in Fig. 1, the difference is considered negligible.

Left with star catalogues we then computed the transformation parameters needed using information from the USNO-B1.0 catalogue (Monet et al. 2003). However, as many of the USNOB1.0 stars were saturated in our images, we extended our reference catalogue by detecting more sources in FITS files derived from the Digitized Sky Survey ${ }^{3}$. We matched the target catalogues to the reference catalogue, solving for a set of low-order spatial polynomials mapping the images onto each other, by repeatedly refining the least squares minimisations using outlier rejection.

\subsubsection{Final master image and object catalogue}

Gain and quantum efficiency variations between chips and differential extinction between exposures were solved for by least squares minimisations. The extinction corrections between the exposures were very small, typically $\sim 0.01 \mathrm{mag}$, whereas the zero-point offsets between the chips were $\sim 0.1 \mathrm{mag}$. As an accurate absolute photometric calibration was not necessary for the present work, we adopted standard Landolt magnitude zeropoints.

The coaddition was done after magnitude corrections were applied to the data. In addition cosmic rays were masked out, before the median image was computed and the background flattened. A master object catalogue was created, where each object's WCS coordinates were calculated from the astrometric solution, and their ellipticity parameters computed (see Sect. 3). Finally we masked out false detections by overplotting the objects onto the image, hence ensuring a final object catalogue free from spurious detections.

${ }^{3}$ http://archive.stsci . edu/dss/index.html

\section{Shear measurements}

Identifying weak lensing effects requires measuring the ellipticities of a large number of faint background galaxies. The main source of noise in weak lensing analysis is the intrinsic ellipticities of these galaxies. To distinguish between distorted images resulting from a weak lens and the usual distribution of shapes existing in an unlensed galaxy population, the ellipticities must be examined for a systematic change. In particular, a tangential alignment of the galaxy shapes around the cluster centre would confirm the existence of a weak lens.

An additional source of error comes from the faint background galaxies being smeared by the PSF, caused by atmospheric turbulence and optical aberrations. The weak shear signal is hence diluted because this smearing will cause the galaxy images to appear more circular than before the smearing. In addition, PSF anisotropies distort the images, causing the galaxies to appear more elliptical, hence introducing false shear signals. It is crucial that these PSF effects are corrected for.

\subsection{PSF corrections}

Following the KSB+ method developed by Kaiser et al. (1995), Luppino \& Kaiser (1997), and Hoekstra et al. (1998), we present a short summary of our implementation below. KSB+ inverts the effects of PSF smearing and anisotropy on objects in an image, presenting a method to recover the true shear.

Ignoring the effects of photon noise, it is possible to express the observed ellipticity of a galaxy as

$e_{\alpha}^{\mathrm{obs}}=e_{\alpha}^{\mathrm{s}}+P_{\alpha \beta}^{\gamma} g_{\beta}+P_{\alpha \beta}^{\mathrm{sm}} p_{\beta}$,

where the first term represents the intrinsic ellipticity of the galaxy, the second term the shift in ellipticity caused by gravitational shear, and the third term the smearing of the galaxy image from the anisotropic PSF (see Luppino \& Kaiser 1997, with additional corrections from Hoekstra et al. 1998 for a thorough deduction of this equation). Present in Eq. (1) are the pre-seeing shear polarisability tensor, $P_{\alpha \beta}^{\gamma}$, and the smear polarisability tensor, $P_{\alpha \beta}^{\mathrm{sm}}$. The latter can be calculated for each object together 
Abell 1351 before correction

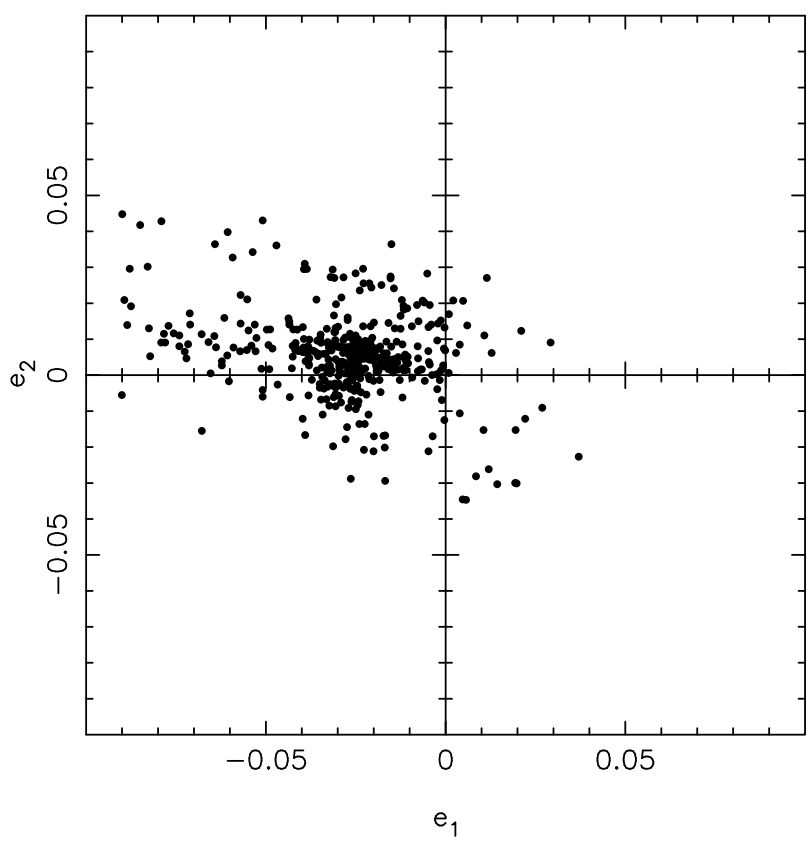

Abell 1995 before correction

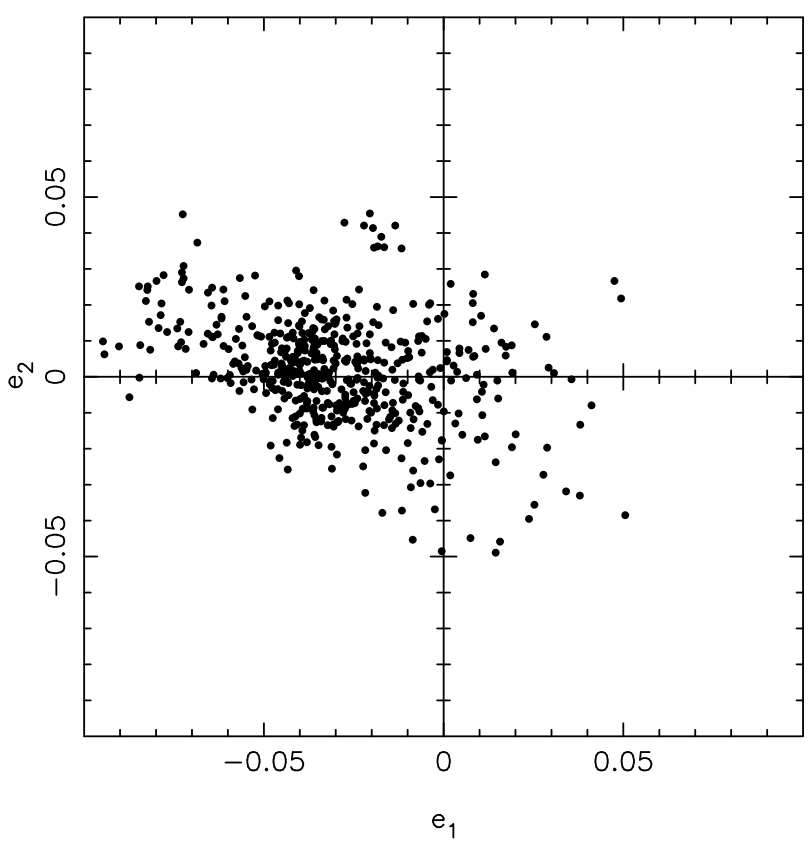

Abell 1351 after correction

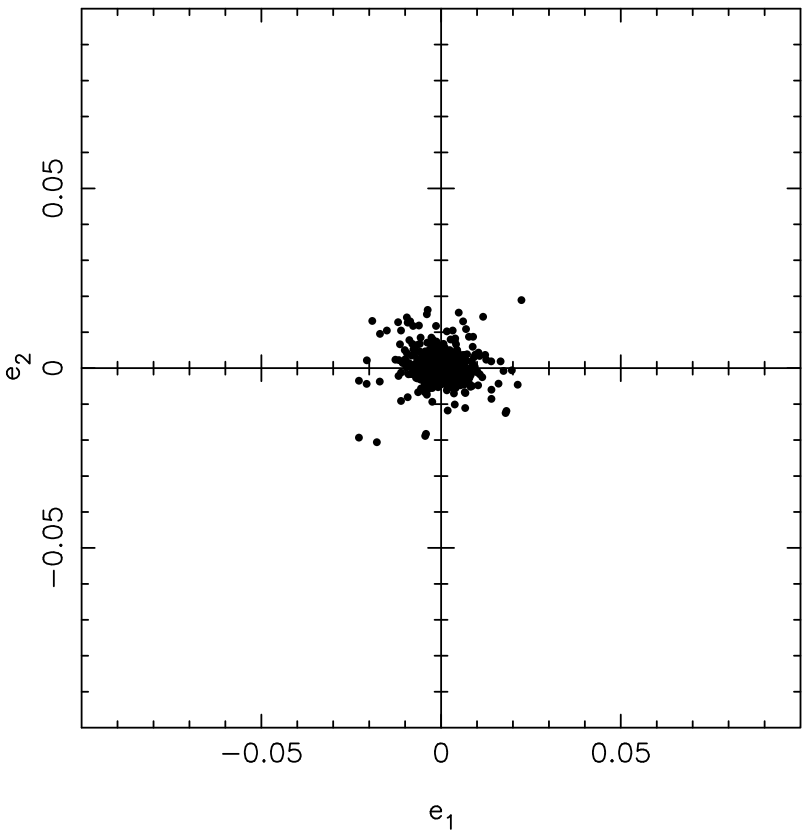

Abell 1995 after correction

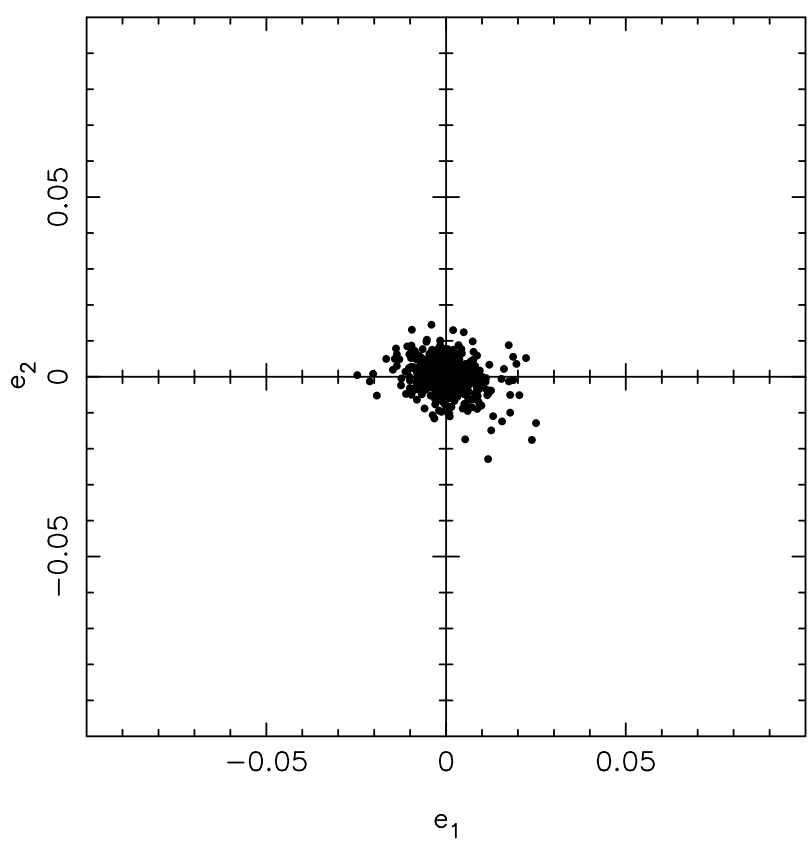

Fig. 2. Ellipticities of the stars in the field of Abell 1351 (top) and Abell 1995 (bottom) before and after corrections for PSF anisotropies. The stars initially had systematic ellipticities up to $\sim 7-9 \%$ in one direction. The PSF corrections reduced these effects to typically $<1.5 \%$.

with $e_{\alpha}^{\mathrm{obs}}$, the post-seeing shear polarisability tensor $P_{\alpha \beta}^{\mathrm{sh}}$, and the centroid of the object.

Because stars are foreground objects $\left(g_{\beta}=0\right)$ and intrinsically circular $\left(e_{\alpha}^{\mathrm{s}}=0\right)$, applying Eq. (1) to stellar objects provides a measure of the total PSF anisotropy, $p_{\beta}$. This was calculated from bright stars selected from the final object catalogue (Sect. 2.2.4). The PSF corrections were then calculated for all individual objects and corrections applied respectively.

The ellipticities of the stars were fitted to a sixth-order Taylor series expansion. When comparing mass and B-mode maps (Sect. 4) for fits of different orders, there was little change with the order of fit. Over the whole field, 410 and 530 stars were used in the fitting process for Abell 1351 and Abell 1995 respectively. Figure 2 shows the ellipticities of the stars before and after PSF corrections.

The pre-seeing shear polarisability tensor, $P_{\alpha \beta}^{\gamma}$, is defined in $\mathrm{KSB}+$ to be

$P_{\alpha \beta}^{\gamma}=P_{\alpha \beta}^{\mathrm{sh}}-P_{\alpha \mu}^{\mathrm{sm}}\left(P^{\mathrm{sm} \star}\right)_{\mu \delta}^{-1} P_{\delta \beta}^{\mathrm{sh} \star}$,

where the asterisk denotes $P_{\alpha \beta}^{\mathrm{sh}}$ and $P_{\alpha \beta}^{\mathrm{sm}}$ applied to stellar objects. From Eq. (1) we see that the reduced shear, $g=\gamma /(1-\kappa)$, is given by

$g_{\beta}=\left(P^{\gamma}\right)_{\alpha \beta}^{-1}\left[e_{\alpha}^{\mathrm{obs}}-P_{\alpha \beta}^{\mathrm{sm}} p_{\beta}\right]$ 
Following the approach by Wold et al. (2002), we assume the PSF is close to circular after the correction, and the polarisabilities can be approximated by $P_{\alpha \beta}=\frac{1}{2}\left(P_{11}+P_{22}\right) \delta_{\alpha \beta}$. The average of $P^{\mathrm{sh} \star} / P^{\mathrm{sm} \star}$ can be calculated as

$\left\langle\frac{P^{\mathrm{sh} \star}}{P^{\mathrm{sm} \star}}\right\rangle=\frac{1}{N_{\mathrm{stars}}} \sum_{\text {stars }} \frac{P_{11}^{\mathrm{sh} \star}+P_{22}^{\mathrm{sh} \star}}{P_{11}^{\mathrm{sm} \star}+P_{22}^{\mathrm{sm} \star}}$,

where using the median value rather than the mean minimises the effect of outliers. Hence Eq. (2) turns into

$P^{\gamma}=\frac{1}{2}\left(P_{11}^{\mathrm{sh}}+P_{22}^{\mathrm{sh}}\right)-\frac{1}{2}\left(P_{11}^{\mathrm{sm}}+P_{22}^{\mathrm{sm}}\right)\left\langle\frac{P^{\mathrm{sh} \star}}{P^{\mathrm{sm} \star}}\right\rangle$.

As $P_{\alpha \beta}^{\text {sh }}$ and $P_{\alpha \beta}^{\text {sm }}$ have already been calculated, this equation is easily solved, and Eq. (3) provides an estimate of the gravitational shear of each object.

Hoekstra et al. (1998) showed that estimating the PSF dilution for each individual galaxy introduces additional noise. We therefore followed their approach by determining $P^{\gamma}$ as a function of magnitude and galaxy size. We determined the median $P^{\gamma}$ within 15 bins in an $r_{g}$-magnitude diagram, where the central 4 bins contain $\sim 4000$ galaxies/bin and the outer ones $\sim 200$ galaxies/bin. We then computed one correction factor for each bin using Eq. (5), and applied this to all galaxies within the corresponding bin.

The faintest and smallest galaxies are more affected by seeing than the larger galaxies, giving them a poorer shape determination and a high correction factor. Such galaxies are therefore of less importance. To account for this, a normalised weight,

$w_{i} \propto\left(\frac{\sigma_{e_{i}}}{\left\langle P^{\gamma}\right\rangle_{i}}\right)^{-2}$,

was calculated for each bin $i$ and assigned to the corresponding galaxies. Here, $\sigma_{e_{i}}$ is the observed dispersion of galaxy ellipticities.

\section{Mass reconstruction}

We selected background galaxies with $6<S / N<100$ for the creation of our mass maps. These maps were constructed from the galaxies' shapes using the finite-field inversion method presented by Seitz \& Schneider (2001; SS01). This method calculates a smoothed shear field on a grid using a modified Gaussian filter. The algorithm then iteratively computes a quantity $K(\theta) \equiv$ $\ln [1-\kappa(\theta)]$ which is determined up to an additive constant due to the mass sheet degeneracy. We could break this degeneracy by assuming that the average convergence vanishes along the border of the wide field of view. The width of the Gaussian term in the filter was set to 2 '.0, resulting in an effective smoothing length of about 1'.6.

In order to evaluate the noise of the mass maps, we computed 2000 mass maps for each cluster based on randomised galaxy orientations, keeping their positions fixed. As the cluster lens signal increases the ellipticities of galaxies, this would lead to an overestimation of the noise at the cluster position. We roughly corrected for this effect by subtracting the expected SIS tangential shear signal, determined from the clusters' known velocity dispersions (see Table 1). Since the singularity of the SIS can lead to overly large corrections close to the cluster centre, we limited the maximum correction factor allowed to 0.5 in each ellipticity component. This affected less than 5 galaxies in both fields. The true mass maps were then divided by the noise maps obtained from the randomised mass maps to create the $S / N$ maps seen in Fig. 3.

Abell 1351 and Abell 1995 are detected with a $S / N$ of 5.3 and 5.2, respectively. Upon integrating the $\kappa$ maps within $r_{200}=$ $1.69 h_{70}^{-1} \mathrm{Mpc}\left(1.50 h_{70}^{-1} \mathrm{Mpc}\right)$ for Abell 1351 (Abell 1995), we find total masses of $M_{2 \mathrm{D}}\left(<r_{200}\right)=11.7 \pm 3.1 \times 10^{14} h_{70}^{-1} M_{\odot}$ and $M_{2 \mathrm{D}}\left(<r_{200}\right)=10.5 \pm 2.7 \times 10^{14} h_{70}^{-1} M_{\odot}$ for the clusters, respectively. The $r_{200}$ radii have been taken from what we consider to be the best NFW fits to the data (see Table 1 and Sect. 5.2), while the errors were determined from integrating the same areas in the 2000 noise maps.

The B-modes in both cluster fields are shown in Fig. 3, computed by repeating the $\kappa$ reconstruction with all galaxies rotated $45^{\circ}$ (Crittenden et al. 2002). Provided that the lensing data are free from systematics and that the noise (intrinsic ellipticities) is Gaussian, these B-mode maps should be consistent with Gaussian noise. Given the effective filter scale of 1'.6, about 380 independent peaks can be placed in the CFH12K field. Thus one would expect 1.1 noise peaks above $3 \sigma$ in the field. A more realistic estimate comes from the 2000 randomisations as these are based on the real ellipticity and spatial distribution. We expect 1.4 (1.6) such peaks for Abell 1351 (Abell 1995). In the real B-mode maps we find 3 peaks for each of the clusters. This is insignificant, as in our randomisations at least 3 such peaks appear per field in $20 \%$ of the cases. In case of Abell 1995 the highest B-mode peak has a significance of 3.9 $\sigma$. Its B-modes appear generally somewhat larger than for Abell 1351, which has no B-mode peaks higher than $3.5 \sigma$.

\subsection{Mass and galaxy density distributions}

In order to compare the surface density maps with the distribution of cluster galaxies, we extracted the red sequence (see e.g. Gladders \& Yee 2000) and investigated the distribution of the selected galaxies. To match the $I$-band and the $V$-band images, we resampled both data sets to the same pixel scale, resulting in a common area of $18.5 \times 18.5$ on the sky. The $V$-band image seeing is around $0 . ' 7$, and thus consistently better than in the $I$-band. The $V$-band data were therefore convolved to match the seeing in the I-band data, 0.' 95 for Abell 1351 and 1'. 15 for Abell 1995. Aperture photometry was carried out using SExtractor (Bertin \& Arnouts 1996) in double-image mode. The deep I-band images served as the detection image, providing us with a target list with defined coordinates. At these positions we integrated the flux in a $3^{\prime \prime}$ wide aperture in each of the $V$ - and $I$-band images. Plotting the galaxies in a colour-magnitude diagram will then in principle provide enough information to separate the red early-type cluster members from the other galaxies.

Each cluster's red sequence did not clearly stand out from the $V-I$ vs. $I$ diagram when all objects were plotted. However, selecting only galaxies within a radius of $3^{\prime}$ of the brightest cluster galaxy (BCG) for the colour-magnitude diagram (see Fig. 4) enabled us to detect a red sequence at $1.4<V-I<1.9$ for both clusters. The selection criteria indicated by the box in each plot were then applied to the entire object catalogue. The number density of the galaxies selected was then calculated as a function of position and overplotted onto the central $9^{\prime}$ of the mass maps (see Fig. 5).

The number density maps were normalised by the fluctuation measured in the field outside the clusters. The centres of Abell 1351 and Abell 1995 are then detected with $15.5 \sigma$ and $9.9 \sigma$ significance, respectively. The positional offsets between mass centres, BCGs, and centres of galaxy density distributions are in the range of $30^{\prime \prime}-55^{\prime \prime}$ for both clusters, and are due 
Table 1. Results from fitting theoretical density profiles to the measured shear values, where each subheadline indicates the cluster centre around which the fitting was done.

\begin{tabular}{|c|c|c|c|c|c|}
\hline BCG & Abell 1351 & Abell 1995 & $\mathbf{S S 0 1}^{a} \kappa$ map & Abell 1351 & Abell 1995 \\
\hline SIS $\left[\chi^{2} /\right.$ d.o.f. $]$ & 1.60 & 1.70 & SIS $\left[\chi^{2} /\right.$ d.o.f. $]$ & 1.44 & 1.14 \\
\hline$\theta_{\mathrm{E}}\left[{ }^{\prime \prime}\right]$ & $16.2_{-2.4}^{+2.3}$ & $12.2 \pm 2.6$ & $\theta_{\mathrm{E}}\left[{ }^{\prime \prime}\right]$ & $15.0_{-2.3}^{+2.5}$ & $12.0_{-2.8}^{+2.5}$ \\
\hline$\sigma_{v}\left[\mathrm{~km} \mathrm{~s}^{-1}\right]$ & $1040_{-80}^{+70}$ & $900_{-100}^{+90}$ & $\sigma_{v}\left[\mathrm{~km} \mathrm{~s}^{-1}\right]$ & $1000 \pm 80$ & $890_{-110}^{+90}$ \\
\hline$M_{\mathrm{SIS}}\left[10^{14} h_{70}^{-1} M_{\odot}\right]$ & $\begin{array}{r}8.7_{-1.4}^{+1.3} \\
\end{array}$ & $5.8_{-1.3}^{+1.2}$ & $M_{\mathrm{SIS}}\left[10^{14} h_{70}^{-1} M_{\odot}\right]$ & $7.8_{-1.4}^{+1.3}$ & $5.5_{-1.4}^{+1.3}$ \\
\hline NFW [ $\chi^{2} /$ d.o.f. $]$ & 1.32 & 1.64 & NFW $\left[\chi^{2} /\right.$ d.o.f. $]$ & 1.12 & 1.11 \\
\hline$c$ & $9.1_{-4.6}^{+\infty}$ & $5.2_{-3.4}^{+\infty}$ & $c$ & $16.1_{-8.4}^{+\infty}$ & $3.0_{-1.9}^{+9.0}$ \\
\hline$r_{200}\left[h_{70}^{-1} \mathrm{Mpc}\right]$ & $1.73_{-0.10}^{+0.13}$ & $1.53_{-0.13}^{+0.17}$ & $r_{200}\left[h_{70}^{-1} \mathrm{Mpc}\right]$ & $1.69_{-0.11}^{+0.10}$ & $1.50_{-0.11}^{+0.20}$ \\
\hline$M_{200}\left[10^{14} h_{70}^{-1} M_{\odot}\right]$ & $8.1_{-1.4}^{+1.8}$ & $5.6_{-1.4}^{+1.9}$ & $M_{200}\left[10^{14} h_{70}^{-1} M_{\odot}\right]$ & $7.5_{-1.5}^{+1.3}$ & $5.3_{-1.2}^{+2.1}$ \\
\hline NFW (fixed $c)\left[\chi^{2} /\right.$ d.o.f. $]$ & 1.42 & 1.66 & NFW (fixed $c$ ) $\left[\chi^{2} /\right.$ d.o.f. $]$ & 1.31 & 1.12 \\
\hline$c$ & 3.7 & 3.7 & $c$ & 3.7 & 3.7 \\
\hline$r_{200}\left[h_{70}^{-1} \mathrm{Mpc}\right]$ & $1.81_{-0.16}^{+0.057}$ & $1.56_{-0.16}^{+0.13}$ & $r_{200}\left[h_{70}^{-1} \mathrm{Mpc}\right]$ & $1.67_{-0.086}^{+0.16}$ & $1.51_{-0.14}^{+0.16}$ \\
\hline$M_{200}\left[10^{14} h_{70}^{-1} M_{\odot}\right]$ & $9.4_{-2.4}^{+0.9}$ & $\begin{array}{c}5.9_{-1.8}^{+1.5} \\
-1.5\end{array}$ & $M_{200}\left[10^{14} h_{70}^{-1} M_{\odot}\right]$ & $7.4_{-1.1}^{+2.1}$ & $5.4_{-1.5}^{+1.7}$ \\
\hline $\mathbf{K S 9 3}^{b} \kappa$ map & & & Galaxy density & & \\
\hline SIS $\left[\chi^{2} /\right.$ d.o.f. $]$ & 1.30 & 2.32 & SIS $\left[\chi^{2} /\right.$ d.o.f. $]$ & 1.17 & \\
\hline$\theta_{\mathrm{E}}\left[{ }^{\prime \prime}\right]$ & $16.5_{-2.4}^{+2.6}$ & $10.4_{-2.6}^{+2.7}$ & $\theta_{\mathrm{E}}\left[{ }^{\prime \prime}\right]$ & $16.5_{-2.4}^{+2.3}$ & \\
\hline$\sigma_{v}\left[\mathrm{~km} \mathrm{~s}^{-1}\right]$ & $1050 \pm 80$ & $830_{-110}^{+100}$ & $\sigma_{v}\left[\mathrm{~km} \mathrm{~s}^{-1}\right]$ & $1050_{-80}^{+70}$ & \\
\hline$M_{\mathrm{SIS}}\left[10^{14} h_{70}^{-1} M_{\odot}\right]$ & $8.9 \pm 1.5$ & $4.9_{-1.4}^{+1.3}$ & $M_{\text {SIS }}\left[10^{14} h_{70}^{-1} M_{\odot}\right]$ & $9.0_{-1.5}^{+1.3}$ & \\
\hline $\mathbf{N F W}\left[\chi^{2} /\right.$ d.o.f. $]$ & 0.96 & 2.22 & NFW $\left[\chi^{2} /\right.$ d.o.f. $]$ & 0.88 & \\
\hline$c$ & $11.2_{-4.9}^{+\infty}$ & $0.9_{-0.4}^{+2.1}$ & $c$ & $11.3_{-6.7}^{+\infty}$ & \\
\hline$r_{200}\left[h_{70}^{-1} \mathrm{Mpc}\right]$ & $1.74_{-0.10}^{+0.11}$ & $1.50_{-0.24}^{+0.17}$ & $r_{200}\left[h_{70}^{-1} \mathrm{Mpc}\right]$ & $1.76_{-0.11}^{+0.10}$ & \\
\hline$M_{200}\left[10^{14} h_{70}^{-1} M_{\odot}\right]$ & $\begin{array}{l}8.3_{-1.4}^{+1.6} \\
\end{array}$ & $\begin{array}{l}5.3_{-2.6}^{+1.8} \\
\end{array}$ & $M_{200}\left[10^{14} h_{70}^{-1} M_{\odot}\right]$ & $8.5_{-1.7}^{+1.5}$ & \\
\hline $\mathbf{N F W}($ fixed $c)\left[\chi^{2} /\right.$ d.o.f. $]$ & 1.13 & 2.33 & NFW (fixed $c$ ) [ $\chi^{2} /$ d.o.f. $]$ & 1.00 & \\
\hline$c$ & 3.7 & 3.7 & $c$ & 3.7 & \\
\hline$r_{200}\left[h_{70}^{-1} \mathrm{Mpc}\right]$ & $1.81_{-0.13}^{+0.10}$ & $1.51_{-0.23}^{+0.11}$ & $r_{200}\left[h_{70}^{-1} \mathrm{Mpc}\right]$ & $1.79_{-0.11}^{+0.10}$ & \\
\hline$M_{200}\left[10^{14} h_{70}^{-1} M_{\odot}\right]$ & $9.4_{-2.0}^{+1.6}$ & $5.4_{-2.5}^{+1.2}$ & $M_{200}\left[10^{14} h_{70}^{-1} M_{\odot}\right]$ & $9.0_{-1.7}^{+1.5}$ & \\
\hline \multicolumn{6}{|l|}{ Integration of SS01 $\kappa$ maps } \\
\hline$r_{200}\left[h_{70}^{-1} \mathrm{Mpc}\right]$ & 1.69 & 1.50 & & & \\
\hline$M_{2 \mathrm{D}}\left(<r_{200}\right)\left[10^{14} h_{70}^{-1} M_{\odot}\right]$ & $11.7 \pm 3.1$ & $10.5 \pm 2.7$ & & & \\
\hline
\end{tabular}

${ }^{a}$ Seitz \& Schneider (2001). ${ }^{b}$ Kaiser \& Squires (1993).

Note that the KSB+PSF correction tends to underestimate the shear by $10-15 \%$, which in turn reduces the cluster masses up to $20 \%$.

to noise in the mass maps. Changing the width of the Gaussian kernel in the finite-field reconstruction algorithm shows that the peak centres can drift by up to $20^{\prime \prime}$ from the mean position. These offsets are consistent with other results in the literature, such as Clowe et al. (2006a), who observed offsets of the order of $10^{\prime \prime}$ between the lensing peaks (of higher $S / N$ than ours) and the optical centres of the bullet cluster. Positional offsets of $50^{\prime \prime}$ are common in the sample of 70 shear-selected clusters by Schirmer et al. (2007).

The cluster galaxy distribution resembles well the mass distribution in the central part of Abell 1351. It extends significantly towards the northeast, a feature also seen in the mass map where we find a local maximum which we refer to as peak $\mathrm{A}$ (see Sect. 4.2). The galaxy distribution of Abell 1995 appears elliptical and elongated in the northeast-southwest direction. This property is not reflected in the mass map where the peak is of rather circular appearance.

To check the integrity of our mass reconstructions further, we applied the peak finder ( $S$-statistics) developed by Schirmer et al. (2007). This method detects areas of enhanced tangential shear using the aperture mass statistics (Schneider 1996). Since it uses a filter function that mimics the tangential shear profile of galaxy clusters it is well suited for detecting mass concentrations. With this method we recover Abell 1351 at the $7.0 \sigma$ level in a $10^{\prime}$ wide filter, and Abell 1995 with $6.1 \sigma$ for a $7^{\prime}$ filter. The filter shape parameter (Schirmer et al. 2007) was chosen as $x_{\mathrm{c}}=0.2$ in both cases. We find that the $S$-statistics is elongated in the same way as the mass reconstruction for Abell 1351, extending towards peak A. We evaluate the significance of this possible substructure in the following.

\subsection{Lower mass peaks in the fields}

In the mass reconstructions two neighbouring peaks $\mathrm{A}$ and $\mathrm{B}$ are detected around Abell 1351, and another one (peak C) in the field of Abell 1995. Their $S / N$-ratios are 4.2, 3.8, and 3.8, respectively. We used the 2000 noise randomisations for each field to find that the probability of a noise peak higher than $4.2 \sigma(3.8 \sigma)$ in the field of Abell 1351 is $0.8 \%(6.8 \%)$, respectively. The corresponding probability for peak $\mathrm{C}$ in the Abell 1995 data is $7.0 \%$. These are somewhat higher than what would be expected from purely Gaussian noise.

Hence the only significant substructure detected in the mass reconstructions is peak A near Abell 1351. Looking at the 


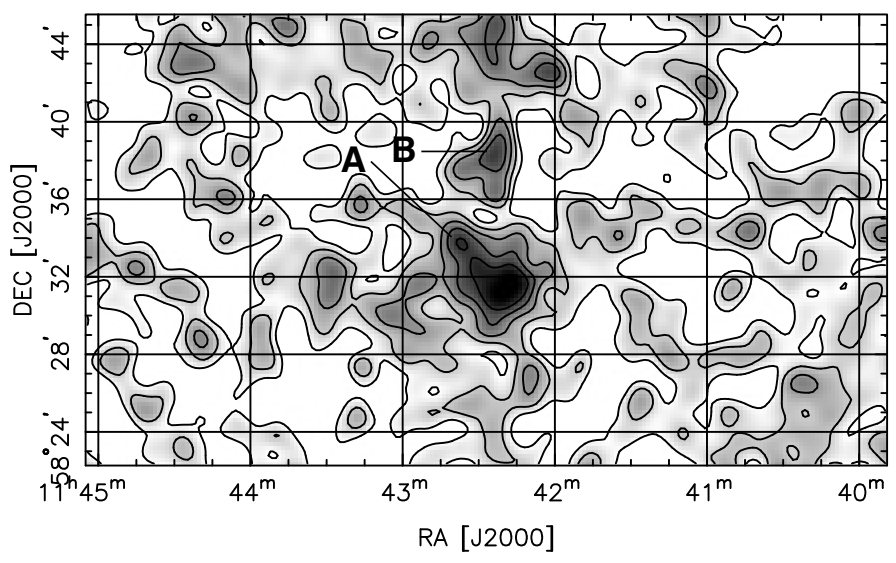

Mass reconstruction of Abell 1995

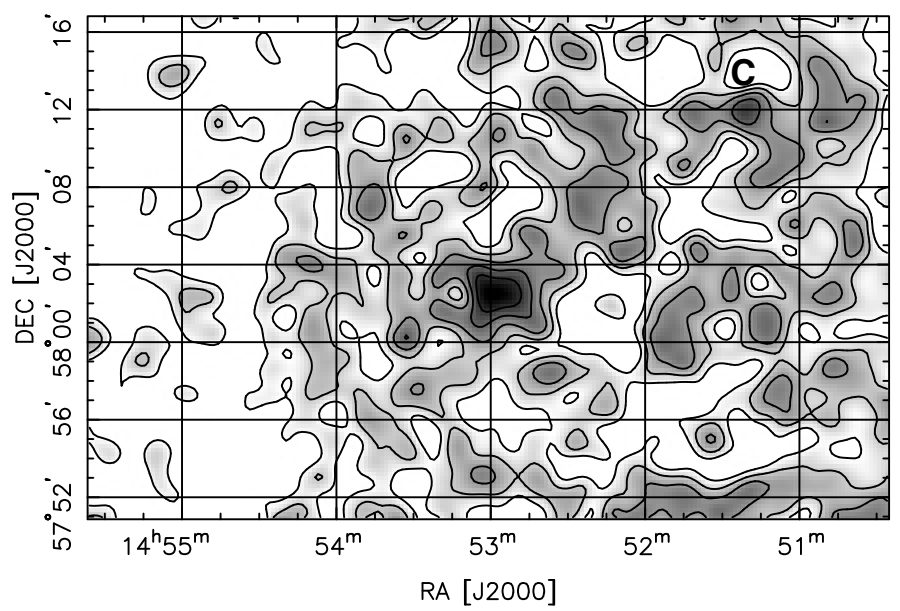

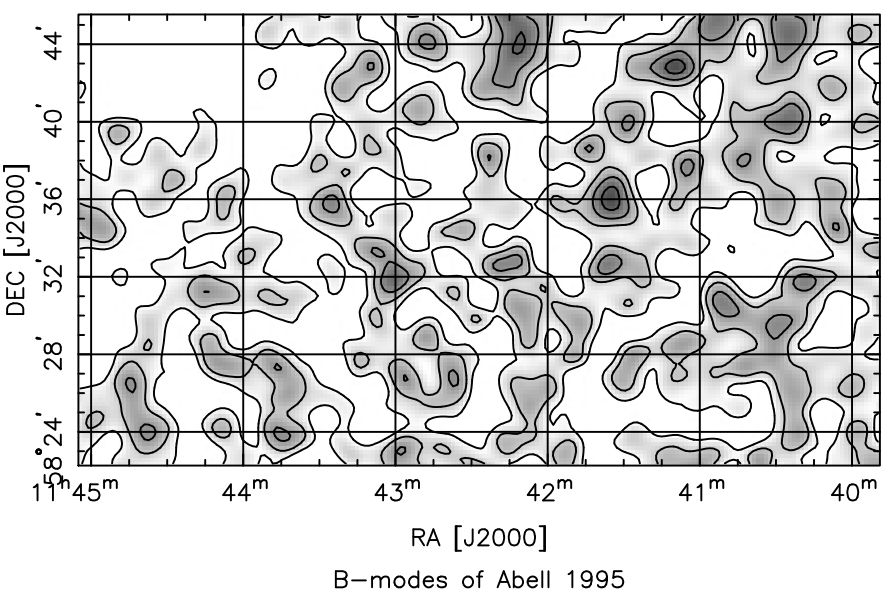

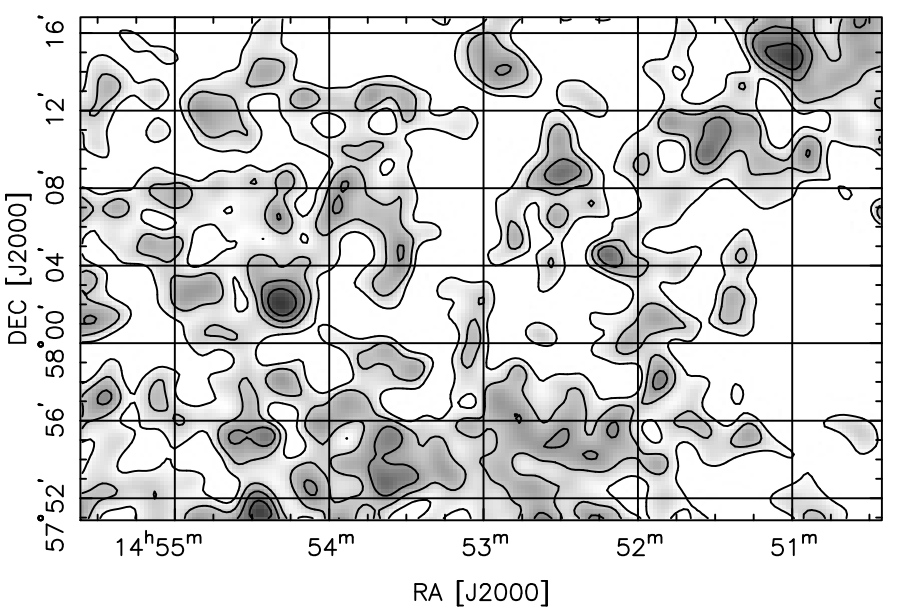

Fig. 3. The projected surface mass densities and B-modes for both clusters in the full CFH12K field, using a finite-field mass reconstruction. The maps show the $S / N$ of the clusters, with contours starting at $0 \sigma$ and increasing in steps of $1 \sigma$. The clusters are detected at significance levels of $5.3 \sigma$ (Abell 1351) and 5.2 $\sigma$ (Abell 1995). The lower peaks A, B, and C have $S / N$-ratios of 4.2, 3.8, and 3.8, respectively. The effective smoothing length of the reconstructions is 1'.6. The highest B-mode peak of Abell 1351 (Abell 1995) has a $S / N$-ratio of 3.5 (3.9).

contours in the upper right panel of Fig. 5, one can see that the $S$-statistics trace this structure as well at the 4.5-5.0 $\sigma$-level. We note that we recovered this substructure over a broad range of filter scales in the $S$-statistics and hence think that it is a real feature in the mass distribution of Abell 1351.

Out of the broad range $\left(1^{\prime}-15^{\prime}\right)$ of filters probed with the $S$-statistics, peak B is detected only once with $4.0 \sigma$ in the $4^{\prime}$ wide filter for $x_{\mathrm{c}}=0.5$. It has the typical characteristics of the dark peaks found by Schirmer et al. (2007), i.e. it is not associated with any overdensity of galaxies. It is therefore most likely a noise peak.

In the Abell 1995 field we could not detect any other peaks using the $S$-statistics. Since the B-modes for those data show a maximum of $3.9 \sigma$ near peak $\mathrm{C}$ (at $3.8 \sigma$ ), we consider it to be a noise peak. As it also lies outside the area covered by the $V$-band, we could not check for overdensities of red galaxies at this position.

\section{Modelling the lensing data}

The mass of a galaxy cluster can be estimated by comparing observed distortions in the background galaxies to those predicted by theoretical density profiles. Using $\chi^{2}$-minimisations of SIS and NFW models we first determined the best fit parameters and then calculated the cluster masses.

The theoretical profiles are both spherically symmetric. We therefore averaged the tangential reduced shear, $g_{\mathrm{t}}=\gamma_{\mathrm{t}} /(1-\kappa)$ (for $r>\theta_{\mathrm{E}}$, where $\theta_{\mathrm{E}}$ is the Einstein radius), in 17 radial bins around the cluster centre. The bins are logarithmically spaced, covering the entire field of view, and starting at $r_{\min }=150^{\prime \prime}$ to avoid the large contamination from cluster galaxies close to the centre of the field (see also Sect. 5.1). To determine the cluster centre, we tested three different positions. First we adopted the peak location in the mass reconstructions generated (Sect. 4). These coincide with the centres of the $S$-statistics. Second the BCG served as cluster centre, and third we tried the centre of the galaxy density of each cluster. As the last coincide with the BCG for Abell 1995, only two positions were tested for this cluster. We also considered strong lensing features, but found that they do not offer further insight in this respect (see Sect. 6.3.1 for details). We calculated $\left\langle g_{\mathrm{t}}\right\rangle_{i}$ for each radial bin $i$ and compared them to the theoretical values at the average radius of each bin, $\langle r\rangle_{i}$.

When calculating the mass of a cluster, the relative distance of the background galaxies and the lensing cluster is required. As we have no specific information about the redshifts of the background galaxies, the distances had to be estimated 

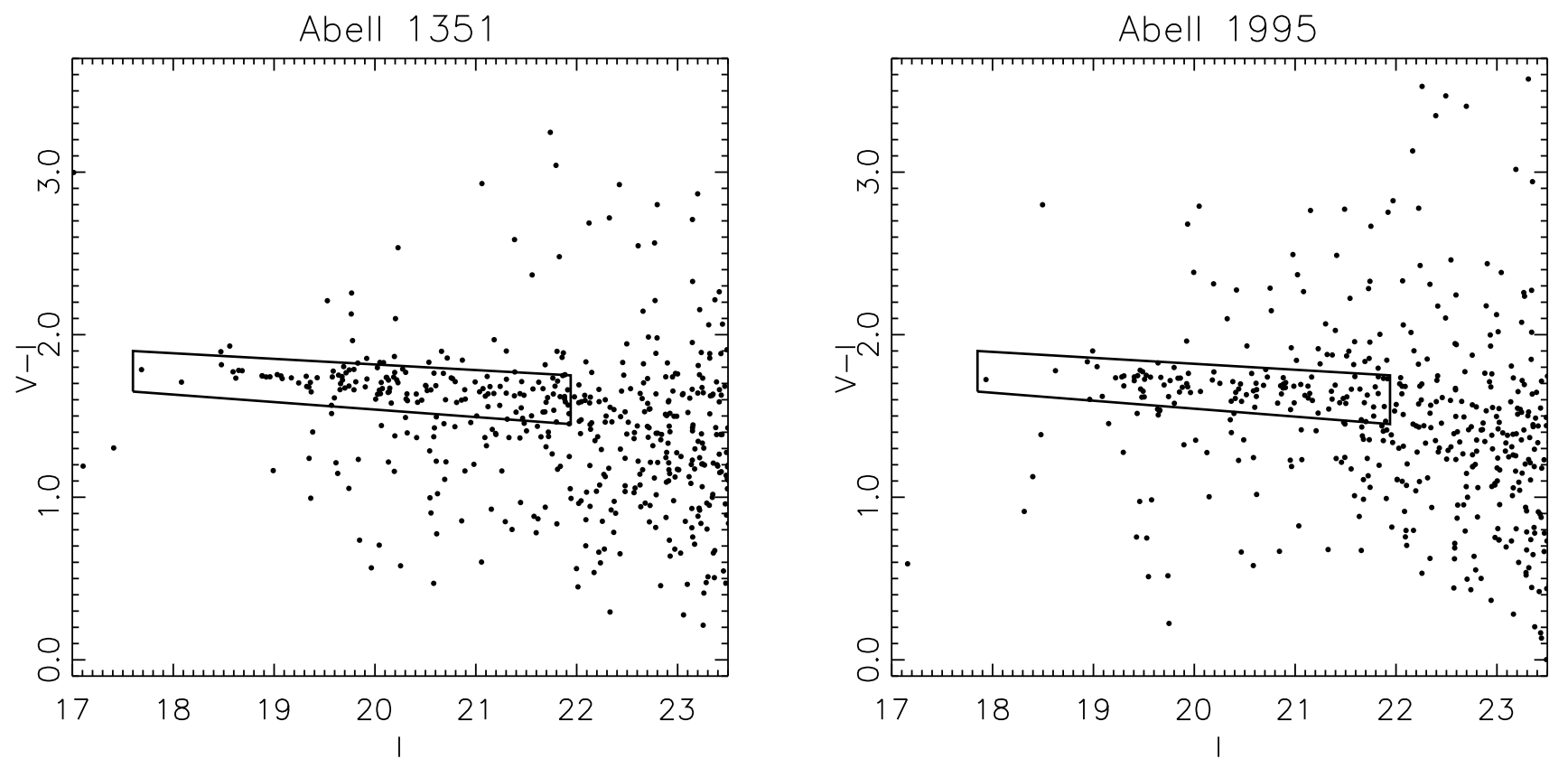

Fig. 4. Colour-magnitude diagrams for Abell 1351 (left) and Abell 1995 (right), where only galaxies within 3' from the BCG are plotted. The red sequence can be seen for $1.4<V-I<1.9$ for both clusters, the box indicating our selection criteria.

statistically. By using the photometric redshift distribution of corresponding faint galaxies from the Hubble Deep Field North (Fernández-Soto et al. 1999), we estimated the average $\beta \equiv D_{\mathrm{ds}} / D_{s}$, where $D_{\mathrm{ds}}$ is the angular diameter distance between the lens and the source and $D_{s}$ between the observer and the source. The empirical relation

$\langle\beta\rangle=-1.21 z_{\mathrm{d}}+0.91$

is derived for a $\Lambda \mathrm{CDM}$ cosmology analogously to the equation of Dahle et al. (2002) for an Einstein-de Sitter universe, which also accounts for the redshift distribution of the source galaxies. Here $z_{\mathrm{d}}$ denotes the redshift of the lens.

\subsection{Cluster contamination and magnification depletion}

At small projected radii from the cluster centre our faint galaxy sample will contain cluster galaxies in addition to background galaxies. We could not discriminate faint cluster members from lensed field galaxies using $V-I$ colours, hence the sample of presumed lensed background galaxies remained contaminated, leading to a systematic bias of the shear measurements towards lower values. In order to quantify this contamination, we determined the overdensity of galaxies in the background catalogue at the cluster position compared to the mean density in the field (an example is shown in Fig. 6 for Abell 1351). A contamination rate of $50 \%$ was found for both cluster centres, vanishing for radii larger than about $4^{\prime}-5^{\prime}$.

The cluster galaxy contamination is in fact even higher than stated above, as magnification depletion leads to a reduced number density of lensed galaxies in the $I$-band near the cluster centre. We found, however, that this effect can be neglected in our case. From the smoothed convergence (see Sect. 4) and reduced shear fields we estimated the magnification using $\mu=$ $((1-\kappa)(1-g))^{-2}$. We found very similar magnifications for both clusters, being 1.65 at the centre and becoming indistinguishable from the noise $\left(\sigma_{\mu} \sim 0.15\right)$ for radii larger than $\sim 3^{\prime}$. The depletion of the number density of lensed galaxies is $\propto \mu^{2.5 s-1}$, with $s=0.15$ in red filters (see e.g. Narayan \& Bartelmann 1996). At the cluster centres the number densities are thus reduced by a factor of $\sim 0.73$, and at a radius of 1.5 magnification depletion becomes indistinguishable from the natural fluctuations in the distribution of field galaxies. Magnification depletion hence only affects the innermost $\sim 0.3 \mathrm{Mpc}\left(100^{\prime \prime}\right)$ of the clusters and can be neglected since we compare the tangential shear profiles to models only for radii larger than $0.5 \mathrm{Mpc}$ (see Fig. 7).

In order to correct for the contamination by cluster galaxies, we modified the theoretical shear values. The reason for adjusting the theoretical values rather than the measured values is that this method is considerably easier to implement. The correction factors were determined in radial bins of logarithmic spacing. One correction value was then calculated for each of the 17 bins in which $\left\langle g_{\mathrm{t}}\right\rangle$ had been measured. By assuming the edges of the field to be approximately free from cluster galaxies, the outermost correction factor could be set to 1 to mimic contaminationfree boundaries of the field. Finally the best fit was found using $\chi^{2}$-minimisations.

\subsection{Fitting the SIS and the NFW profiles}

Once the cluster centre is determined, the only free parameter of the SIS profile is the velocity dispersion, $\sigma_{v}$. The best fit of the SIS profile is determined by $\chi^{2}$-minimisation for a range of $\sigma_{v}$ values, the results being shown in Table 1. The mass estimate, $M_{\text {SIS }}$, for this profile is calculated at $r_{200}$ (the radius inside which the mean mass density of the cluster equals $200 \rho_{\text {crit }}$ ) found in the NFW fitting with two free parameters utilising the same cluster centre.

The NFW profile is derived from fitting the density profiles of numerically simulated cold dark matter halos. It appears to give a very good description of the radial mass distribution inside the virial radius of a galaxy cluster. For a thorough introduction 
Abell 1351 (mass + num. density)

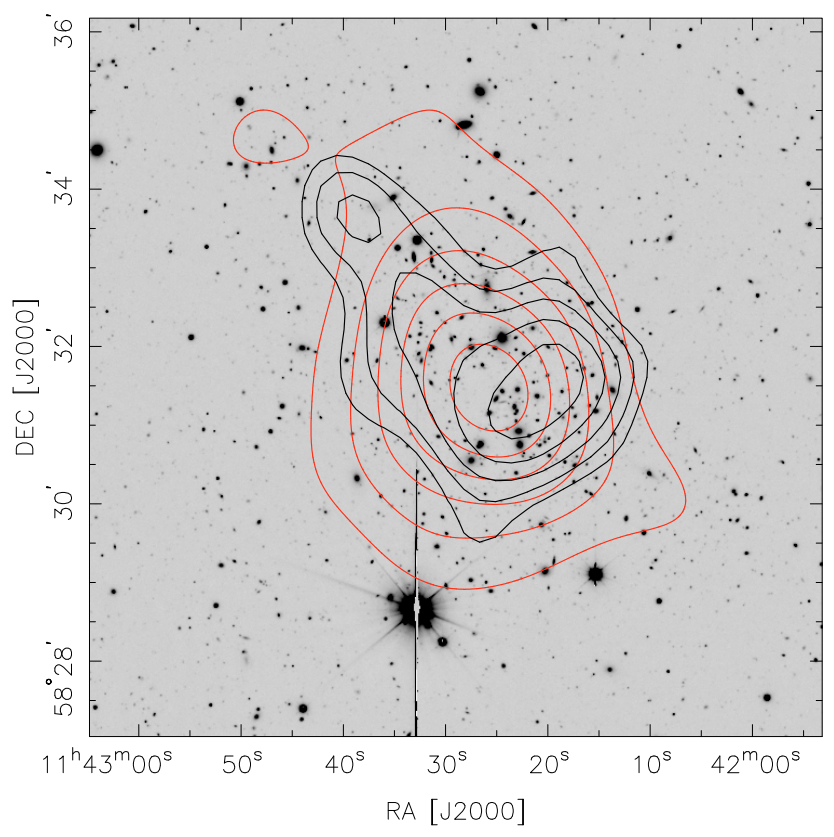

Abell 1995 (mass + num. density)

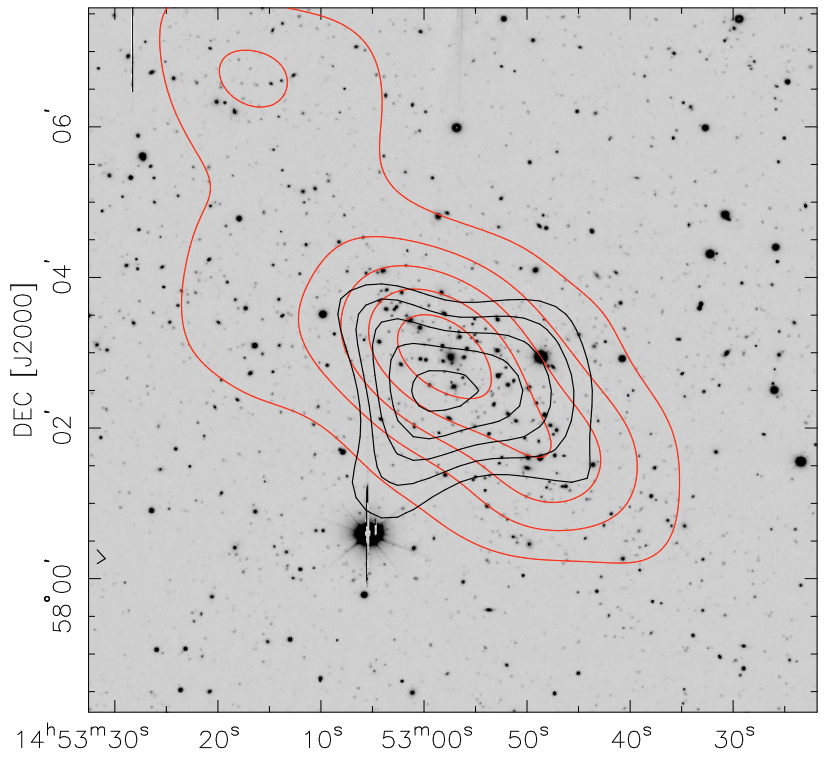

RA [J2000]
Abell 1351 (S-statistics + num. density)

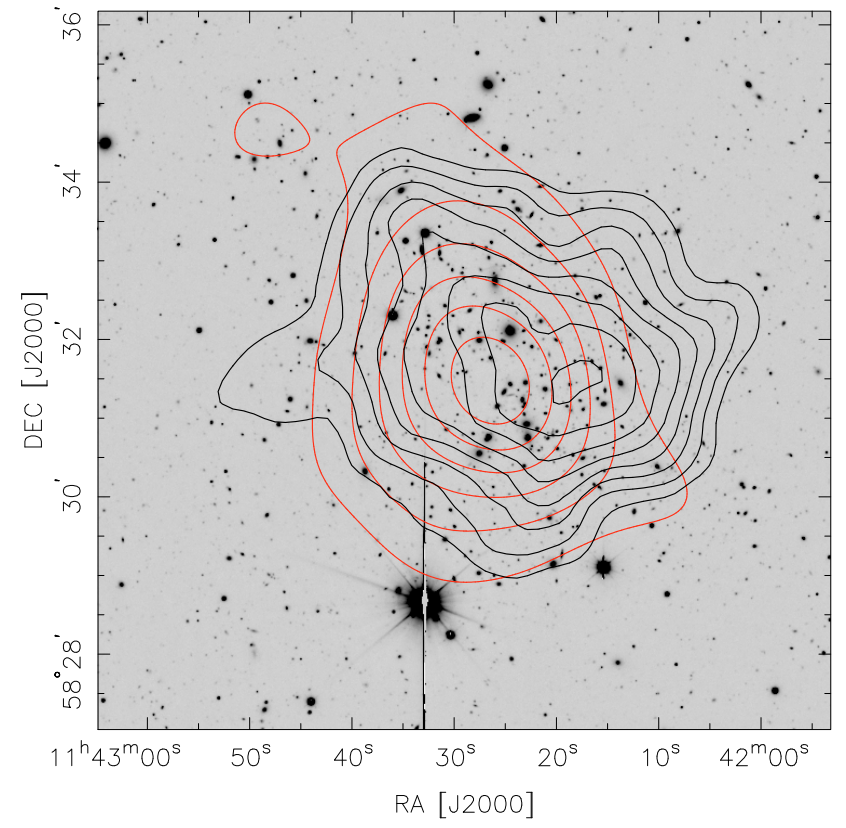

Abell 1995 (S-statistics + num. density)

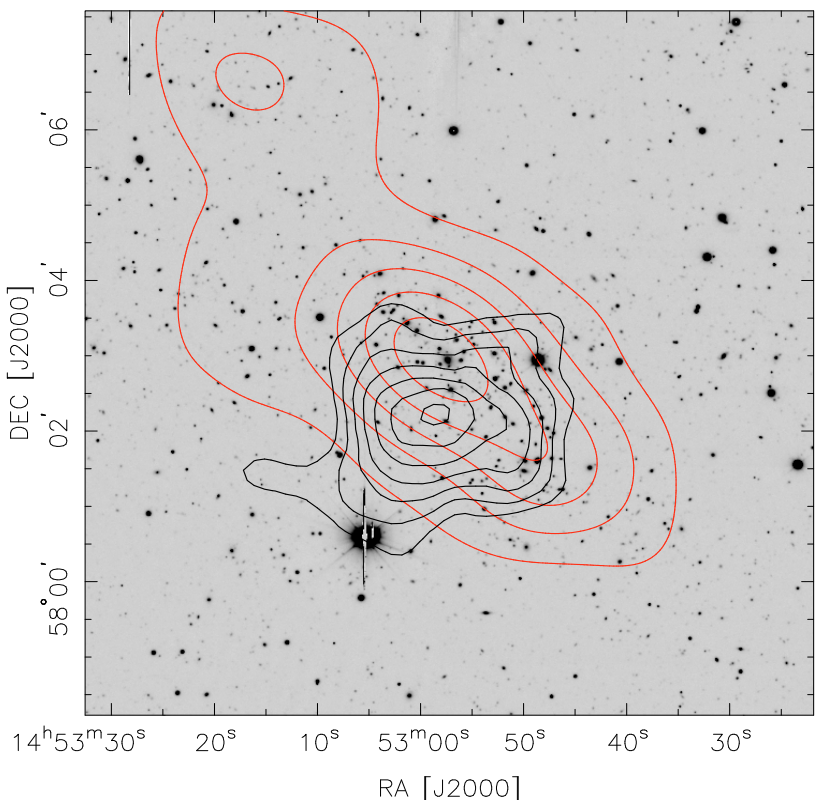

Fig. 5. The black contours show the mass reconstruction (left) and S-statistics (right) for Abell 1351 (top) and Abell 1995 (bottom). The contours start at the $3.0 \sigma$ level and increase in steps of $0.5 \sigma$. Overplotted in white contours (red in online version) are the number densities of galaxies selected with the red sequence method, normalised by the rms fluctuation in the field outside the clusters. These contours are isodensity contours, starting at $3 \sigma$ going in steps of $3 \sigma$, and peak at $15 \sigma$ and $10 \sigma$, respectively.

to the gravitational lensing properties of the NFW mass density profile we refer the reader to Wright \& Brainerd (2000). The theoretical $\gamma_{\mathrm{t}}$ and $\kappa$ can be calculated analytically for the NFW density profile (Bartelmann \& Schneider 2001). We derived the best fit parameters for different values of the concentration parameter, $c$, ranging from 0.1 to 24.9 in steps of 0.1 .

With the cluster centre fixed, the NFW profile has two free parameters, $r_{200}$ and $c$. We fitted our shear measurements to this profile twice; first by keeping $c$ fixed and varying only $r_{200}$ to find our best fit, and second by varying both parameters. The best fit parameters were determined by minimising $\chi^{2}$ in both cases. Based on N-body simulations of dark matter halos, Bullock et al. (2001) derived relations for the mean value of $c$ as a function of redshift and mass for different cosmologies. For a halo of mass $M_{\mathrm{vir}}=8 \times 10^{14} M_{\odot}$, the relation yields

$$
c=\frac{5.8}{1.194\left(1+z_{\mathrm{d}}\right)}
$$

(where $r_{200}=1.194 r_{\text {vir }}$ for a $\Lambda$ CDM cosmological model). As this mass is close to the mass estimates of Abell 1351 and 
Cluster galaxy contamination in faint galaxy sample

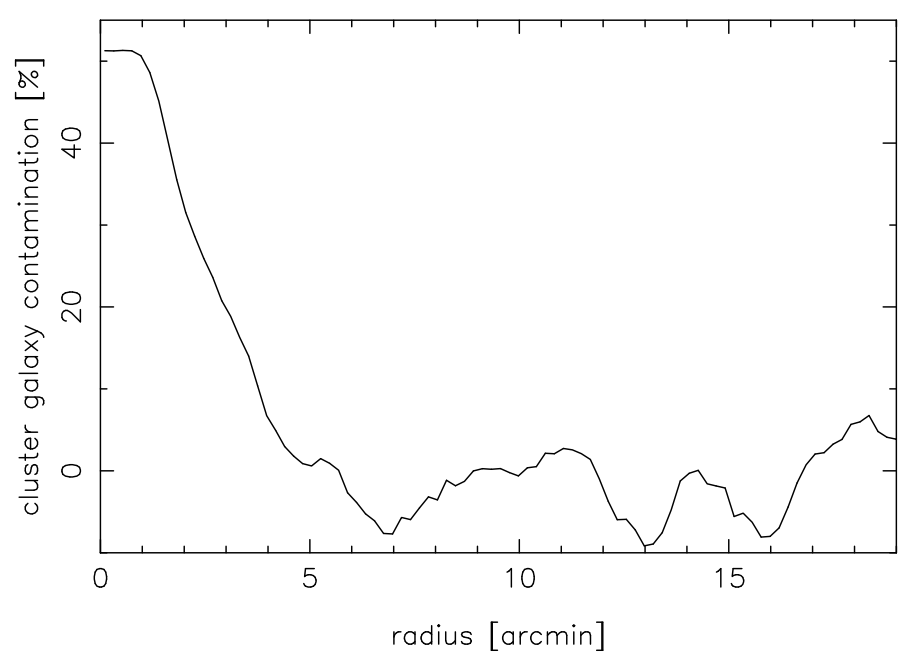

Fig. 6. Percentage of cluster galaxies in the faint galaxy catalogue of Abell 1351 (that of Abell 1995 is very similar). Because the projected density of cluster galaxies is assumed to equal zero at the edge of the field, the cluster galaxy contamination was set to zero here by subtracting the median value outside the central area of the image (the large field of view makes this a well-working approximation). Our fitting procedure starts at $r_{\min }=150^{\prime \prime}$ to avoid the large cluster galaxy contamination at the centre.

Abell 1995 (Sect. 4), the weak mass dependence of $c$ can be disregarded. In the second fitting process both $r_{200}$ and $c$ were altered, creating a grid of $c, r_{200}$-values. The best fit $r_{200}$ was first determined for each value of $c$, then the best fit $c$ was found. The results are given in Table 1 . We could not find an upper limit for the concentration parameter of Abell 1351, independent of the cluster centre chosen. The same holds for Abell 1995 in case the $\mathrm{BCG}$ is chosen as the centre. We discuss this in more detail in Sect. 6.2.

As an example we display the reduced tangential shear as a function of radius using the SS01 $\kappa$ maps as cluster centre, see the left diagrams of Fig. 7. The measured values of $\left\langle g_{t}\right\rangle$ are given together with the best fit models of the SIS and NFW profiles. Judging from the diagrams alone, the NFW profile letting both $c$ and $r_{200}$ vary seems to represent the best fit to the clusters. However, the $\chi^{2} /$ d.o.f. values given for each model in Table 1 show that the differences between the models are not statistically significant. The differences emerging from different cluster centres seem to have a higher impact. The right diagrams of Fig. 7 show the B-modes of both clusters, i.e. the cross-component of $\left\langle g_{\mathrm{t}}\right\rangle$ as a function of radius. Both measurements are consistent with zero.

\section{Discussion}

X-ray studies show Abell 1351 to be a galaxy cluster exhibiting significant dynamical activity and undergoing a major merger event (Allen et al. 2003), which indicates a cluster still in its formation phase. Analyses assuming a relaxed cluster will hence naturally differ from weak lensing analyses, where no assumption is made about the dynamical state of the cluster. One example is the virial analysis by Irgens et al. (2002), where an unusually high velocity dispersion of $\sigma_{v}=1680_{-229}^{+340} \mathrm{~km} \mathrm{~s}^{-1}$ was obtained for Abell 1351, based on radial velocity measurements of 17 cluster galaxies. Such a high velocity dispersion is not uncommon in merging systems. If for example two smaller clusters with low velocity dispersions fall towards each other along the line of sight with a velocity comparable to or higher than their $\sigma_{v}$, then a very large total $\sigma_{v}$ would be inferred, with a correspondingly overestimated virial mass. The cluster CL0056.03 - 37.55 is a good example for such a system (see Schirmer et al. 2003).

Abell 1995 is, unlike Abell 1351, classified as a relaxed cluster in dynamical equilibrium (Pedersen \& Dahle 2007). X-ray studies and virial analyses of this cluster are hence also more compatible with lensing studies (Patel et al. 2000; Irgens et al. 2002). The projected two-dimensional distribution of cluster galaxies in Abell 1995 is clearly elliptical (see Fig. 5), whereas the central lensing mass distribution is circular.

\subsection{The mass estimates}

The mass distributions of Abell 1351 and Abell 1995 were estimated assuming that the clusters follow spherically symmetric SIS or NFW profiles. Although an elliptical mass profile might yield more accurate cluster mass estimations, Dietrich et al. (2005) showed that the results from fitting a singular isothermal ellipse model depend strongly on the initial values chosen for the minimisation routines. We therefore decided not to fit this profile to our clusters.

Heymans et al. (2006) demonstrated in the shear testing programme that the KSB+ PSF correction tends to systematically underestimate the shear values $\sim 10-15 \%$. To measure how much this affects our data, we calculated an upper limit for our mass estimates by increasing the ellipticities with $15 \%$ and repeating the fitting process. We found that the underestimation of shear leads to an underestimation of the total cluster mass with a maximum of $20 \%$, which is within the initial error bars. The concentration parameters did not change significantly by this boosting of ellipticities. Since we do not know by exactly how much our shear values are underestimated this was merely an attempt to quantify this effect on our data, and is not taken into account in the results presented in this paper.

Dahle et al. (2002) obtained weak lensing estimates of the cluster velocity dispersion of several clusters using an SIS model and assuming an Einstein-de Sitter universe. Their results were $\sigma_{v}=1410_{-90}^{+80} \mathrm{~km} \mathrm{~s}^{-1}$ for Abell 1351 and $\sigma_{v}=1240 \pm 80 \mathrm{~km} \mathrm{~s}^{-1}$ for Abell 1995, and do not agree with our results. However, there are several important differences in methodology between Dahle et al. (2002) and our work. As mentioned above, the assumed cosmological model is different. Also, the first paper approximated $g_{\mathrm{t}}=\gamma_{\mathrm{t}}$, whereas we use $g_{\mathrm{t}}=\gamma_{\mathrm{t}} /(1-\kappa)$ in our fits and mass reconstructions. Finally, the shear estimator of Kaiser (2000) used by Dahle et al. (2002) is shown by Heymans et al. (2006) to have a nonlinear response to shear. A re-analysis of the Dahle et al. (2002) data, taking all these effects into account, yielded new values of $\sigma_{v}=1410 \pm 90 \mathrm{~km} \mathrm{~s}^{-1}$ and $\sigma_{v}=1000 \pm 100 \mathrm{~km} \mathrm{~s}^{-1}$ for Abell 1351 and Abell 1995, respectively. Hence there still remains a systematic discrepancy between the results for Abell 1351, while the measurements for Abell 1995 agree within error bars.

A remaining difference between our work and Dahle et al. (2002) is the maximum radius, $r_{\max }$, to which the shear is measured, given by the field of view of the detector. Changing $r_{\max }$ in our Abell 1351 data to 550" (as this is the value used by Dahle et al. 2002) led to $\sigma_{v}=1240 \pm 105 \mathrm{~km} \mathrm{~s}^{-1}$, consistent with the re-analysed Dahle et al. (2002) values within error bars. 


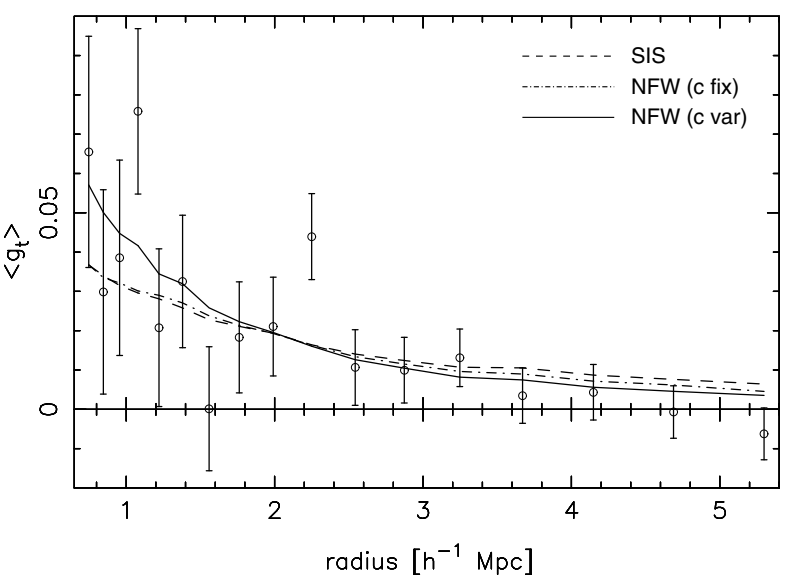

Average tangential distortion of Abell 1995

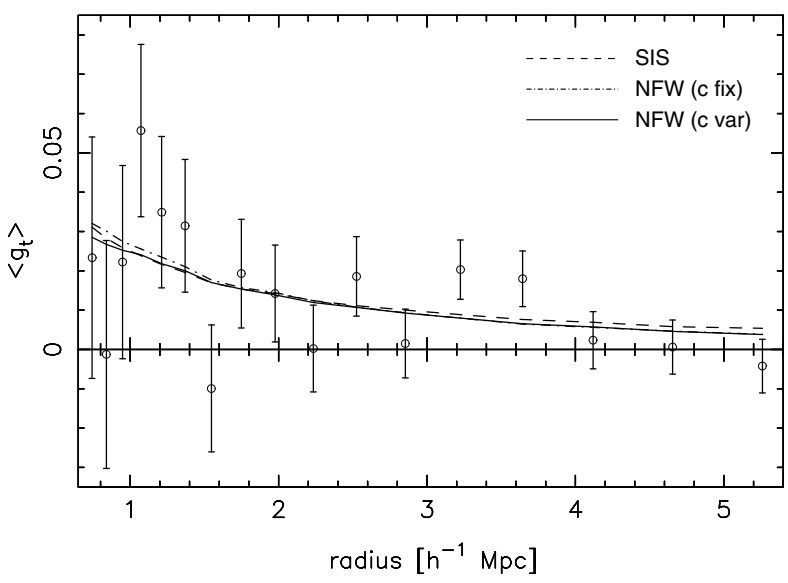

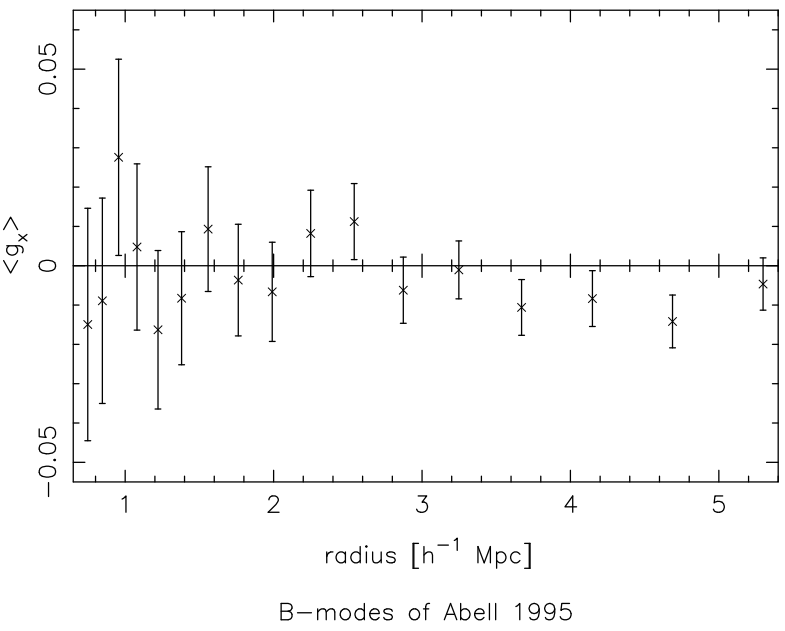

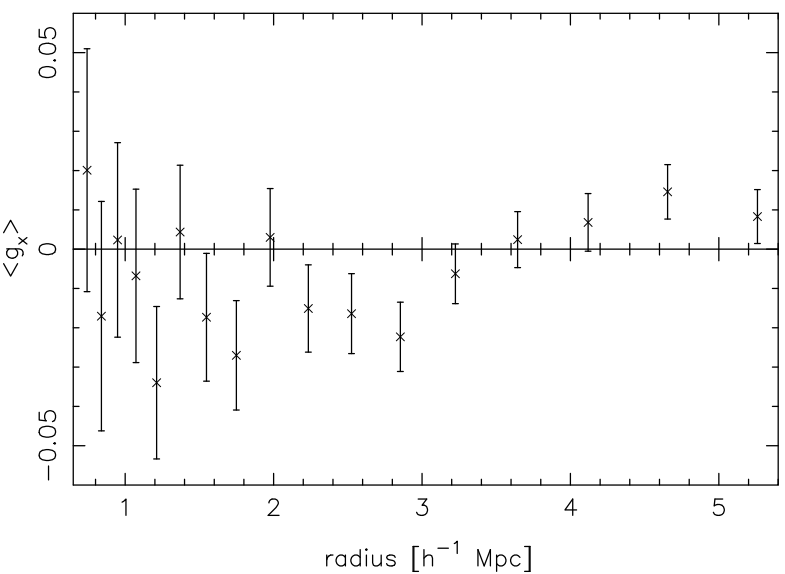

Fig. 7. Left: Reduced tangential shear as a function of radius for Abell 1351 (top) and Abell 1995 (bottom) using the SS01 $\kappa$ map as cluster centre (the other centres show very similar figures). The averaged gravitational lensing distortions of background galaxies are shown as points with $1 \sigma$ error bars. The lines indicate the best fit models; the dashed line representing the SIS profile, the dot-dashed line the NFW profile keeping $c$ fixed, and the solid line the NFW profile with two free parameters. It should be noted that these lines represent the theoretical values after modifications from cluster galaxy contamination are applied to each bin independently of each other (see Sect. 5). Right: cross-component of the reduced tangential shear as a function of radius for Abell 1351 (top) and Abell 1995 (bottom). This signal should disappear if $\left\langle g_{\mathrm{t}}\right\rangle$ is caused by lensing only, and it is seen that the measurements are close to zero for both clusters.

Allen et al. (2003) used the Dahle et al. (2002) observations to obtain a weak lensing mass estimate applying the NFW model to a $\Lambda$ CDM cosmology. Their results gave $M_{200}=30.2_{-4.9}^{+5.6} \times 10^{14} h_{70}^{-1} M_{\odot}$ for Abell 1351 and $M_{200}=14.4_{-3.0}^{+3.3} \times 10^{14} h_{70}^{-1} M_{\odot}$ for Abell 1995. These values are high compared to the results of this study. Allen et al. (2003) used a fixed concentration parameter in the fitting process, $c=5$. Applying this value to our data yielded minimal changes in $M_{200}$. The discrepancies hence originate from Allen et al. (2003) utilising $r_{200}=2.69_{-0.19}^{+0.14} h_{70}^{-1} \mathrm{Mpc}$ and $r_{200}=2.07_{-0.14}^{+0.19} h_{70}^{-1} \mathrm{Mpc}$ (priv. comm.) for Abell 1351 and Abell 1995, respectively, as these values are larger than our best fit $r_{200}$ values.

\subsection{The concentration parameter}

The mass density of a cluster with a low concentration parameter decreases slower when going to larger radii than for a cluster with a high $c$ value (Wright \& Brainerd 2000). Although unconstrained upwards, we find a lower limit of $c \geq 4.5$ for Abell 1351. As is also seen from the radial dependence of the shear in Fig. 7 (top left), the mass distribution of Abell 1351 concentrates around the cluster centre, indicating that its concentration parameter is significantly higher than that of Abell 1995. The values found for $c$ of Abell 1995 (see Table 1) suggest that its mass is spread more evenly to larger radii, which is also seen in Fig. 7 (bottom left).

From their aperture mass calculations Dahle et al. (2002) found that most of the mass of Abell 1995 is contained within $r \sim 0.9 h_{70}^{-1} \mathrm{Mpc}\left(\sim 200^{\prime \prime}\right)$. The mass of Abell 1351 shows the opposite behaviour, increasing evenly with radius, even at large radii. These results are contrary to our conclusions. As measurements at large radii are certain to include additional information not recognised close to the cluster centre, these discrepancies are likely explained by the difference in field-size between the two studies. By mapping the mass distribution towards a radius more than twice the size as that of Dahle et al. (2002), our results are better constrained. Further bias also arises from the measurements of Dahle et al. (2002) starting from an inner radius of $r_{\text {min }}=50^{\prime \prime}\left(\sim 0.37 h_{70}^{-1} \mathrm{Mpc}\right)$, where we consider the cluster galaxy contamination to be very high, in addition to not including any correction for this contamination. 
Table 2. Results from varying the inner radius from where the shear values of Abell 1351 are measured.

\begin{tabular}{ccccccc}
\hline \hline $\begin{array}{c}r_{\min } \\
{\left[{ }^{\prime \prime}\right]}\end{array}$ & $\begin{array}{c}r_{s} \\
{\left[{ }^{\prime \prime}\right]}\end{array}$ & $c$ & $\begin{array}{c}r_{200} \\
{\left[h_{70}^{-1} \mathrm{Mpc}\right]}\end{array}$ & $\begin{array}{c}M_{200} \\
{\left[10^{14} h_{70}^{-1} M_{\odot}\right]}\end{array}$ & $\begin{array}{c}\text { No. of } \\
\text { galaxies }\end{array}$ & $\chi^{2} /$ d.o.f. \\
\hline 100 & $76_{-35}^{+56}$ & $4.9_{-2.2}^{+3.6}$ & $1.76_{-0.13}^{+0.057}$ & $8.5_{-1.9}^{+0.8}$ & 15630 & 1.11 \\
110 & $61_{-29}^{+48}$ & $6.0_{-2.8}^{+4.7}$ & $1.71 \pm 0.10$ & $7.9_{ \pm} 1.4$ & 15582 & 1.45 \\
120 & $54_{-26}^{+60}$ & $6.8_{-3.3}^{+7.6}$ & $1.71_{-0.086}^{+0.10}$ & $7.9_{-1.2}^{+1.4}$ & 15529 & 1.31 \\
130 & $60_{-26}^{+83}$ & $6.2_{-2.5}^{+8.5}$ & $1.76_{-0.17}^{+0.057}$ & $8.5_{-1.7}^{+0.8}$ & 15482 & 1.33 \\
140 & $37_{-20}^{+\infty}$ & $10.0_{-5.5}^{+\infty}$ & $1.73_{-0.13}^{+0.11}$ & $8.1_{-1.8}^{+1.6}$ & 15428 & 1.16 \\
150 & $33_{-15}^{+\infty}$ & $11.2_{-4.9}^{+\infty}$ & $1.74_{-0.10}^{+0.11}$ & $8.3_{-1.4}^{+1.6}$ & 15358 & 0.96 \\
\hline
\end{tabular}

Our shear values are measured from a radial cut-off, $r_{\min }=150^{\prime \prime}$, to avoid the large cluster galaxy contamination present at small radii. Because $c$ is estimated from the scale radius, $r_{\mathrm{s}}=r_{200} / c$, it is desirable to include $r_{\mathrm{s}}$ in the measurements $\left(r_{\min }<r_{\mathrm{s}}\right)$ in order to obtain an accurate estimate of the concentration parameter. If this is not the case, $c$ is basically unconstrained.

This appears to be the case for Abell 1351, explaining why we were not able to derive an upper limit for its concentration parameter. Letting $r_{\min }=150^{\prime \prime}$, we ensured a cluster galaxy contamination $<25 \%$ at this inner radius. However, as the $c$ parameter appears unconstrained under this condition, we reduced $r_{\text {min }}$ in an attempt to obtain clearer results. The problem then arising was the increasing contamination of cluster galaxies. Looking at Fig. 6 we see that at $r=120^{\prime \prime}$ the cluster contamination is $\sim 32 \%$, and at $r=100^{\prime \prime}$ it equals $\sim 40 \%$. Though this contamination is accounted for during the fitting process, the contamination correction is still vulnerable to fluctuations in the projected galaxy density caused by foreground and/or background structures.

Table 2 presents the results from letting $100^{\prime \prime} \leq r_{\min } \leq 150^{\prime \prime}$ for Abell 1351 (with the Kaiser \& Squires $1993 \kappa$ map peak as cluster centre, see Sect. 6.3). It is seen that whilst $c$ is decreasing with smaller $r_{\min }, r_{200}$ and $M_{200}$ remain stable for different $r_{\min }$. Also worth noticing is that for $r_{\min } \leq 130^{\prime \prime}, c$ becomes constrained. However, as $r_{\min }>r_{\mathrm{s}}$ for the different starting radii, we cannot obtain further conclusions from these results. As $r_{\mathrm{s}}$ is even smaller for Abell 1995, we did not repeat this test for the cluster. Dietrich et al. (2005) experienced similar problems when attempting to determine the concentration parameter for Abell 222 and Abell 223, concluding that obtaining a reliable $c$ from weak lensing data only is difficult, if not impossible.

\subsubsection{Best fit concentration parameter}

Bullock et al. (2001) presented dark matter halo simulations, attempting to find a "best fit concentration parameter" applicable to all types of halos. They found that for halos of the same mass, the concentration, $c_{\text {vir }} \equiv r_{\text {vir }} / r_{s}$, can be given by $c_{\text {vir }} \propto\left(1+z_{\mathrm{d}}\right)^{-1}$. This is contrary to earlier beliefs that $c_{\text {vir }}$ does not vary much with redshift. Numerically simulated massive clusters typically have concentration parameters 4-5 (Bullock et al. 2001). This is within the limiting values for both clusters, although looking at Fig. 7, the outcome from varying $c$ seems to better follow the shear values of Abell 1351.

There exists several examples of high concentration parameters in the literature. Kneib et al. (2003) found $c=22_{-5}^{+9}$ for the central mass concentration of the cluster $\mathrm{Cl} 0024+1654$. Gavazzi (2005) concluded on $c=11.73 \pm 0.55$ for MS2137-23, while Broadhurst et al. (2005) found $c=13.7_{-1.1}^{+1.4}$ for Abell 1689. Limousin et al. (2007) presented a thorough discussion of the different concentration parameters derived for Abell 1689 in the literature, concluding that a distribution of best fit $c$ parameters is needed for observed lensing clusters in order to provide a sample large enough to make an adequate comparison with simulations. A recent study of observed concentration values for clusters by Comerford \& Natarajan (2007) show that the best fit lensing-derived $c$ parameters are systematically higher than concentrations derived via $\mathrm{X}$-ray measurements, a difference which can be at least partly explained by effects of triaxiality of cluster halos (Corless \& King 2007; Gavazzi 2005; Oguri et al. 2005; Clowe et al. 2004) or the substructure within the clusters (King \& Corless 2007), although the latter effect may also produce a negative bias of $c$ values. In addition, baryonic physics can increase the concentration parameter mildly by up to $10 \%$ as compared to dissipationless dark matter in pure dark matter simulations (see e.g. Lin et al. 2006).

\subsection{Centre position}

In addition to the three centre positions tested in Sect. 5, we computed $\kappa$ maps with the inversion method of Kaiser \& Squires (1993; KS93) and utilised the peak of this surface mass distribution as a fourth cluster centre. The KS93 method assumes that $\gamma=g$, which is not a good approximation near the centres of massive systems. Therefore, in comparison with the other methods, it provided us with a reference point as for how large variation one can reasonably expect for the various centroiding methods.

All centre positions obtained with the four methods lie within $1^{\prime}$ and hence represent the errors expected when using the peak of a $\kappa$ map as cluster centre. As can be seen from Table 1, varying the centre position only slightly can lead to different mass estimates. Although within error bars, the results from fitting NFW using a fixed $c$ varies most. The NFW fitting of two parameters is more stable with a smaller spread in $M_{200}$. This is also reflected in $\chi^{2} /$ d.o.f., as a value closer to 1 is a better fit.

Worth noticing is the generally smaller differences between the results of Abell 1995 as compared to those of Abell 1351. The concentration parameter also seems better constrained for Abell 1995, where we could not obtain an upper limit for $c$ only in the case where the BCG was used as the centre reference. On the other hand, an upper limit for $c$ could not be obtained for Abell 1351 for any of the cluster centres chosen. This is consistent with the fact that Abell 1351 is not in dynamical equilibrium, lacking a well-defined cluster centre. The results obtained from fitting spherically symmetric models hence depend on the cluster centre chosen.

\subsubsection{Strong lensing features}

Strong lensing effects are in general susceptive to substructures in clusters, and might constrain the centre of mass further. For 
both clusters recent archival WFPC2 HST data exists, taken for an ongoing snapshot survey of X-ray luminous clusters (HST PID 11103, PI: H. Ebeling). The images are taken through the F606W filter totalling 1200 s exposure time each.

Taking into account both the colours and morphologies of galaxies in our $V-I$ data and the morphologies in the HST images, there are at least half a dozen plausible arcs and arclets visible in each of the two clusters. The lensing pattern for Abell 1351 appears to be very complex and does not indicate a single, welldefined centre. This is supported by the presence of several elliptical galaxies which are of similar brightness as the BCG. On the contrary, for Abell 1995 several arc(let)s are well aligned around the BCG (apart from three which are obviously associated with individual cluster galaxies), justifying adopting the BCG as cluster centre for Abell 1995. Applying strong lensing to our data will therefore not offer further constraints on the determination of the centre of mass than we already have.

\subsection{The mass reconstructions}

In Sect. 4 we presented the weak lensing reconstruction of the clusters' surface mass density, detecting the clusters on the $5 \sigma$ level. In case of Abell 1351 a significant neighbouring peak A was detected, which coincides with the galaxy distribution.

We note significant differences comparing our mass maps to those of Dahle et al. (2002), who used the KS93 inversion method. Abell 1995 appears rather circular in both reconstructions, with attached filamentary structures of low significance seen in the KS93 map. In their re-analysis of the Abell 901 supercluster field, Heymans et al. (2008) argued that such filamentary structures could be enhanced by the KS93 method itself. This algorithm assumes $g=\gamma$ near the critical cluster cores, which hence overestimates $\kappa$, and the smoothing implemented could then lead to the apparent merging of neighbouring peaks.

Our map of Abell 1351 appears roughly circular at the position of the main cluster with a significant extension towards the northeast, whereas it shows an extension to the southwest in the work of Dahle et al. (2002). Both reconstructions have peak B in common. Since it is not associated with any overdensity of galaxies but appears in both reconstructions based on very different data sets, the most likely explanation is a chance alignment of galaxies triggering this detection.

\section{Conclusions}

Utilising observations from $\mathrm{CFH} 12 \mathrm{~K}$ we find the masses of Abell 1351 (Abell 1995) to be $M_{200} \equiv M\left(r_{200}\right) \sim 8-9 \times$ $10^{14} h_{70}^{-1} M_{\odot}\left(M_{200} \sim 5-6 \times 10^{14} h_{70}^{-1} M_{\odot}\right)$. These results are derived from fitting our data to the NFW profile, altering both $c$ and $r_{200}$, to find the best theoretical fit to our shear measurements (Fig. 7). Our $\kappa$ reconstructions yield mass estimates of $M_{2 \mathrm{D}}\left(<r_{200}\right)=11.7 \pm 3.1 \times 10^{14} h_{70}^{-1} M_{\odot}\left(M_{2 \mathrm{D}}\left(<r_{200}\right)=\right.$ $10.5 \pm 2.7 \times 10^{14} h_{70}^{-1} M_{\odot}$ ) for Abell 1351 (Abell 1995).

Our results illustrate that using solely weak lensing measurements, with no photometric or spectroscopic information to separate cluster members from background galaxies, the concentration parameter found for a galaxy cluster is poorly constrained. Future spectroscopic redshift measurements and strong lens modelling of the arcs seen towards the cores of Abell 1351 and Abell 1995 might help improve the constraints on their concentration parameters. However, the change in $c$ value resulting from small variations of the centre position of Abell 1351 indicates that the mass distribution in the core of this dynamically unrelaxed cluster is too complex to be meaningfully fit by the NFW mass density profile. $M_{200}$, on the other hand, varies only slightly for both clusters when either $r_{\min }$ is decreased or the cluster centre varied. Although the KSB+ PSF correction leads to an underestimation of the cluster masses (with a maximum of $20 \%$ in our case), increasing the background galaxy ellipticities correspondingly still yields masses within error bars of our current estimates. We therefore conclude that the mass estimates presented are robust.

Acknowledgements. The authors thank the anonymous referee for very helpful critics, and Thomas Erben and Peter Schneider for useful and constructive comments. We also thank Aleksi Halkola for helpful discussion during the NFW analysis. K.H. gratefully acknowledges support from a doctoral fellowship awarded by the Research council of Norway, project number 177254/V30.

\section{References}

Allen, S. W., Schmidt, R. W., Fabian, A. C., \& Ebeling, H. 2003, MNRAS, 342, 287

Bartelmann, M., \& Schneider, P. 2001, Phys. Rep., 340, 291

Bertin, E., \& Arnouts, S. 1996, A\&AS, 117, 393

Broadhurst, T., Takada, M., Umetsu, K., et al. 2005, ApJ, 619, L143

Bullock, J. S., Kolatt, T. S., Sigad, Y., et al. 2001, MNRAS, 321, 559

Clowe, D., De Lucia, G., \& King, L. 2004, MNRAS, 350, 1038

Clowe, D., Bradač, M., Gonzalez, A. H., et al. 2006a, ApJ, 648, L109

Clowe, D., Schneider, P., Aragón-Salamanca, A., et al. 2006b, A\&A, 451, 395

Comerford, J. M., \& Natarajan, P. 2007, MNRAS, 379, 190

Corless, V. L., \& King, L. J. 2007, MNRAS, 380, 149

Crittenden, R. G., Natarajan, P., Pen, U.-L., \& Theuns, T. 2002, ApJ, 568, 20

Dahle, H., Kaiser, N., Irgens, R. J., Lilje, P. B., \& Maddox, S. J. 2002, ApJS, 139,313

Dahle, H., Pedersen, K., Lilje, P. B., Maddox, S. J., \& Kaiser, N. 2003, ApJ, 591, 662

Dietrich, J. P., Schneider, P., Clowe, D., Romano-Díaz, E., \& Kerp, J. 2005, A\&A, 440, 453

Fernández-Soto, A., Lanzetta, K. M., \& Yahil, A. 1999, ApJ, 513, 34

Gavazzi, R. 2005, A\&A, 443, 793

Gavazzi, R., \& Soucail, G. 2007, A\&A, 462, 459

Gladders, M., \& Yee, H. 2000, AJ, 120, 2148

Heymans, C., Van Waerbeke, L., Bacon, D., et al. 2006, MNRAS, 368, 1323

Heymans, C., Gray, M. E., Peng, C. Y., et al. 2008, MNRAS, 385, 1431

Hildebrandt, H., Erben, T., Dietrich, J. P., et al. 2006, A\&A, 452, 1121

Hoekstra, H., Franx, M., Kuijken, K., \& Squires, G. 1998, ApJ, 504, 636

Irgens, R. J., Lilje, P. B., Dahle, H., \& Maddox, S. J. 2002, ApJ, 579, 227

Kaiser, N. 2000, ApJ, 537, 555

Kaiser, N., \& Squires, G. 1993, ApJ, 404, 441

Kaiser, N., Squires, G., \& Broadhurst, T. 1995, ApJ, 449, 460

King, L., \& Corless, V. 2007, MNRAS, 374, L37

Kneib, J.-P., Hudelot, P., Ellis, R. S., et al. 2003, ApJ, 598, 804

Limousin, M., Richard, J., Jullo, E., et al. 2007, ApJ, 668, 643

Lin, W. P., Jing, Y. P., Mao, S., Gao, L., \& McCarthy, I. G. 2006, ApJ, 651, 636

Luppino, G. A., \& Kaiser, N. 1997, ApJ, 475, 20

Mahdavi, A., Hoekstra, H., Babul, A., Balam, D. D., \& Capak, P. L. 2007, ApJ, 668,806

Maturi, M., Schirmer, M., Meneghetti, M., Bartelmann, M., \& Moscardini, L. 2007, A\&A, 462, 473

Miyazaki, S., Hamana, T., Ellis, R. S., et al. 2007, ApJ, 669, 714

Monet, D. G., Levine, S. E., Canzian, B., et al. 2003, AJ, 125, 984

Narayan, R., \& Bartelmann, M. 1996 [arXiv:astro-ph/9606001v2]

Navarro, J. F., Frenk, C. S., \& White, S. D. M. 1995, MNRAS, 275, 720

Navarro, J. F., Frenk, C. S., \& White, S. D. M. 1997, ApJ, 490, 493

Oguri, M., Takada, M., Umetsu, K., \& Broadhurst, T. 2005, ApJ, 632, 841

Patel, S. K., Joy, M., Carlstrom, J. E., et al. 2000, ApJ, 541, 37

Pedersen, K., \& Dahle, H. 2007, ApJ, 667, 26

Schirmer, M., Erben, T., Schneider, P., et al. 2003, A\&A, 407, 869

Schirmer, M., Erben, T., Hetterscheidt, M., \& Schneider, P. 2007, A\&A, 462, 875

Schneider, P. 1996, MNRAS, 283, 837

Seitz, S., \& Schneider, P. 2001, A\&A, 374, 740

Wittman, D., Dell'Antonio, I. P., Hughes, J. P., et al. 2006, ApJ, 643, 128

Wold, M., Lacy, M., Dahle, H., Lilje, P. B., \& Ridgway, S. E. 2002, MNRAS, 335,1017

Wright, C. O., \& Brainerd, T. G. 2000, ApJ, 534, 34 